\title{
Molar Binding Energy of Zigzag and Armchair Single-Walled Boron Nitride Nanotubes
}

\author{
Levan Chkhartishvili ${ }^{1}$, Ivane Murusidze ${ }^{2}$ \\ ${ }^{1}$ Department of Physics, Georgian Technical University, Tbilisi, Georgia; ${ }^{2}$ Institute of Applied Physics, Ilia State University, Tbilisi, \\ Georgia. \\ Email: chkharti2003@yahoo.com
}

Received August $4^{\text {th }}, 2010$; revised August $27^{\text {th }}, 2010$; accepted September $6^{\text {th }}, 2010$.

\begin{abstract}
Molar binding energy of the boron nitride single-walled zigzag and armchair nanotubes is calculated within the quasi-classical approach. We find that, in the range of ultra small radii, the binding energy of nanotubes exhibit an oscillatory dependence on tube radius. Nanotubes $(1,1),(3,0)$, and $(4,0)$ are predicted to be more stable species among single-walled boron nitride nanotubes. The obtained binding energies of BN single-walled nanotubes corrected with zero-point vibration energies lies within the interval (12.01-29.39) eV. In particular, molar binding energy of the ultra-large-radius tube is determined as $22.95 \mathrm{eV}$. The spread of the molar zero-point vibration energy of BN nanotubes itself is (0.25-0.33) $\mathrm{eV}$ and its limit for ultra-large-radius tubes is estimated as $0.31 \mathrm{eV}$. The binding energy peak located at 2.691 A corresponds to the equilibrium structural parameter of all realized stable BN nanotubular structures.
\end{abstract}

Keywords: Binding Energy, Zigzag and Armchair Nanotubes, BN

\section{Introduction}

Boron nitride with the chemical formula $\mathrm{BN}$ can be found in the form of one-dimensional diatomic molecules, two-dimensional nanotubes and fullerenes, three-dimensional crystals like the layered hexagonal $\mathrm{h}-\mathrm{BN}$ and rhombohedral $\mathrm{r}-\mathrm{BN}$ as well as turbostratic t-BN, cubic zinc-blende c-BN and wurtzite $\mathrm{w}-\mathrm{BN}$ modifications as well as their nanostructures etc. Boron and nitrogen atoms are surrounded tetrahedrally in both denser c-BN and $\mathrm{w}-\mathrm{BN}$ crystals.

Any constituent atom of an h-BN crystal, which is believed to correspond to the boron nitride ground state, may be considered as a 3-coordinated atom because the strong chemical binding (covalent with some deal of ionic) occurs only within the layers, while weak van der Waals forces seem to be responsible mostly for interlayer binding. The h-BN crystal has a "graphitic" structure with a two-layer stacking sequence (r-BN is characterized by a three-layer stacking). These layers consist of regular hexagons (i.e., 6-membered atomic rings) with vertexes alternatively occupied by $\mathrm{B}$ and $\mathrm{N}$ atoms. In the h-BN crystal, $\mathrm{B}$ atoms are placed directly above $\mathrm{N}$ atoms and vice versa. Therefore one might suppose that the ionicity contributes to interlayer bonding as well. How- ever, actually, the electrostatic component is insignificant due to the large interlayer distances. It is argued also by the existence of a layered r-BN crystal, in which each subsequent layer is turned around an angle of $\pi / 3$, and also by the isolated plane defects and their bundles included in real h-BN crystals, in which any given atom can be placed above the same atom. In addition, it is possible to obtain turbostratic t-BN and amorphous structures in the form of mixes of various boron nitride crystalline phases, and multi-walled nanotubular and multishelled fullerene-like BN structures. Strong chemical bonding between atoms in a given layer and weak interlayer interaction in layered boron nitrides specify an opportunity of physical and chemical intercalations by various atoms and molecules.

Based upon the similarity of structures of the boron nitride layered phases with graphite, it was assumed [1] that along with carbon $\mathrm{C}$ nanotubes, stable $\mathrm{BN}$ nanotubes - fragments of hexagonal or mixed BN layers wrapped into cylinders - could also exist.

Indeed, by means of arc discharge BN nanotubes had been obtained both from carbon nanotubes [2] and in carbon-free plasma [3]. The arc discharge methods were used to produce $\mathrm{B}_{x} \mathrm{C}_{y} \mathrm{~N}_{z}$ nanotubular structures identified by the high-resolution transmission-electron-microscopy 
(TEM) together with $K$-edge electron-energy-loss-spectrometry (EELS) determining the local atomic composition, while, in a carbon-free plasma discharge area between a BN-packed W-rod and a cooled Cu-electrode, multi-walled pure BN tubes were produced with inner diameters on the order of 1 to $3 \mathrm{~nm}$ and with lengths up to $200 \mathrm{~nm}$; EELS of individual tubes yielded $\mathrm{B} / \mathrm{N}$ ratio of approximately 1 .

At present various methods of synthesis of the $\mathrm{BN}$ nanotubular structures are developed.

The arc discharge in a molecular nitrogen atmosphere between electrodes made of graphite and refractory boron compounds, e.g., hafnium diboride $\mathrm{HfB}_{2}$, forms BN-C nanotubes with strong phase separation between $\mathrm{BN}$ and $\mathrm{C}$ layers along the radial direction $[4,5]$. If both electrodes are made of $\mathrm{HfB}_{2}$ rods, single- or double-walled chemically pure $\mathrm{BN}$ nanotubes are formed with a structure close to stoichiometric $\mathrm{B} / \mathrm{N} \sim 1$ [6]. Most obtained tube ends are closed by flat layers perpendicular to the tube axis. A closure by a triangular facet resulting from three $120^{\circ}$-disclinations was proposed to account for this specific shape. For the most part, the multi-walled BN nanotubes are formed with electrodes made of zirconium diboride $\mathrm{ZrB}_{2}[7,8]$. In this case most of the nanotubes have diameters from 3 to $40 \mathrm{~nm}$ and lengths on the order of $100 \mathrm{~nm}$. Single-layer tubes with diameters of 2 to 5 $\mathrm{nm}$ are also formed rarely. The morphology of the tube tips suggests the presence of pentagons and heptagons which are energetically less favorable compared with squares.

A laser melting of the solid-state BN (of any crystal structure, not only layered but also amorphous) at high nitrogen pressure, (5-15) GPa, forms nanotubes free from inclusions, containing from 3 up to 8 walls, and having a characteristic outer diameter of (3-15) $\mathrm{nm}$ [9].

$\mathrm{BN}$ nanotubes have been also obtained by pyrolysis of the molecular precursor with the use of Co catalysts [10].

Bundles of single-walled (or containing few layers) BN nanotubes with almost stoichiometric structure can be formed in substitution reactions - by thermal treatment of a mixture of boron trioxide or trichloride, and bundles of single-walled C-nanotubes at high temperatures, $(1250-1350)^{\circ} \mathrm{C}$, in a nitrogen flow $[11,12]$.

$\mathrm{BN}$ nanotubes also grow in solid-state process that involves neither deposition from the vapor phase nor chemical reactions [13]. The nanotubes were produced by first ball-milling of the layered h-BN powder to generate highly disordered or amorphous nanostructures and followed by the product annealing at temperatures up to $1300^{\circ} \mathrm{C}$. The annealing leads to the nucleation and growth of hexagonal BN nanotubes both of cylindrical and bamboo-like morphologies.

Multi-walled BN nanotubes have been obtained by carbothermal reduction of the ultra-dispersive amorphous boron oxide $\mathrm{B}_{2} \mathrm{O}_{3}$ at simultaneous nitriding at high temperatures, $(1100-1450)^{\circ} \mathrm{C}[14,15]$. For large tubes, it is found that the ratio of length to radius is preserved.

Besides of arc-melting, pyrolysis, and chemical reactions, boron nitride nanotubular structures were created by means of ballistic nuclear displacements caused in a h-BN layered crystal structure by electron irradiation in TEM [16,17].

High growth temperatures (above $1100^{\circ} \mathrm{C}$ ), a low production yield, and impurities have prevented progress in applications of BN nanotubes in the past decade. Rather recently, it has been shown that these tubes can be grown on substrates at lower temperatures (of about $600^{\circ} \mathrm{C}$ ) [18]. High-order tubular structures were constructed, which can be used without further purification.

For synthesizing BN nanotubular material, some methods were inspired from carbon. In particular, these include techniques such as laser ablation and non-ablative laser heating [19]. Transformation of the compressed powders of the fine-grained h-BN into nanotubular form can be induced [20] by the concentrated light energy in nitrogen flow. Fiber-like clusters synthesized by evaporation of the layered $\mathrm{BN}$ in a nitrogen atmosphere and obtained in powders formed on substrate or chamber surfaces contained nanotubes with diameters and lengths equal to (0.05-200) and (100-3000) $\mu \mathrm{m}$, respectively. Applying TEM, there were obtained their associations in tree- and coral-like aggregates.

$\mathrm{BN}$ nanotubes can be grown from a nanococoon seed as well [21].

Recently the development of a new method for producing long, small-diameter, single- and few-walled, BN nanotubes in macroscopic quantities has been reported [22]. The pressurized vapor/condenser (PVC) method produces highly crystalline, very long, small-diameter nanotubes without catalysts. Their palm-sized, cottonlike masses of raw material were grown by this technique and spun directly into centimeters-long yarn. Nanotube lengths were observed to be $\sim 100$ times that of those grown by the most closely related method.

Soon after synthesizing the first BN nanotubes, it was proposed a number of their possible applications in technique [3]. For example, a system of the collinear BN nanotubes forms a boron nitride fiber. At the same time, the theory [23] developed for structural and electronic properties of nanotubular heterojunctions, in which one of the layers is nanotubular boron nitride (namely, for $\mathrm{C} / \mathrm{BN}$ and $\mathrm{BC}_{2} \mathrm{~N} / \mathrm{BN}$ systems), leads to a conclusion that on basis of it a different electronic devices can be designed. In particular, nanostructures with C-layers both in the center and at the periphery separated by a few BNlayers may allow the creation of sandwich nanotubular 
devices [4]. Within the special semiempirical approach [24] C/BN superlattices and isolated junctions have been investigated as specific examples by the wide variety of electronic devices that can be realized using such nanotubes. The bottom of the conduction bands in pure BN nanotubes is controlled by a nearly-free-electron-state localized inside the tube suggesting interesting electronic properties under doping.

Other opportunities of application of the BN nanotubes are connected with the features of their phonon spectrum [25]. Such dielectric tubes without inversion center can be used as a phonon laser in $\mathrm{GHz}-\mathrm{THz}$ range or hypersound quantum generator. Because of the presence of special nanotubular oscillatory modes, there is a strong enhancement of electron-phonon interaction in comparison with a bulk material. It is not excluded that closepacked one-dimensional $\mathrm{BN}$ nanotubes will serve as high-temperature superconductors. The $\mathrm{GHz}$ oscillatory behavior of double-walled $\mathrm{BN}$ nanotubes was also predicted [26,27]. This system can also be employed for making good shock absorbers because application of low pressure leads to its significant compression.

The band gap progression with BN nanotube diameter (which is of crucial importance for device applications) was presented and analyzed in detail in [28]. In zigzag BN nanotubes, radial deformations that give rise to transverse pressures decrease the gap from 5 to $2 \mathrm{eV}$, allowing for optical applications in the visible range [29]. Importantly, both the zigzag and armchair tubes are found [30] (see also [31]) to exhibit large second-order nonlinear optical behavior with the second-harmonic generation and linear electro-optical coefficients being up to 15-times larger than that of bulk BN in both denser zinc-blende and wurtzite structures. This indicates that BN nanotubes are promising materials for nonlinear optical and optoelectronic applications.

The electronic structure of BN nanotubes can be tuned within a wide range through covalent functionalization [32] (see also [33]). The ultraviolet (UV) and visible absorption spectra indicate that their electronic structure undergoes drastic changes under functionalization. First principle calculations revealed that the covalently functionalized BN nanotubes can be either n- or p-doped depending on the electronegativity of molecules attached. Their energy gap can be adjusted from UV to visible optical range by varying concentration of functionalizing species.

One-dimensional crystals of potassium halides, including $\mathrm{KI}, \mathrm{KCl}$, and $\mathrm{KBr}$, were inserted into $\mathrm{BN}$ nanotubes [34]. High-resolution TEM and energy-dispersive X-ray spectrometry were used to characterize their microstructures and compositions. The fillings are usually single crystals with lengths up to several $\mu \mathrm{m}$. The wetting properties (static contact angles of the liquids and surface tension) of individual $\mathrm{BN}$ nanotubes were studied [35] experimentally using a nanotube-based force to measure the interactions between nanotubes and liquids in situ.

First principles simulations on the interaction of molecular hydrogen $\mathrm{H}_{2}$ with the native and substitutional defects in small-diameter $(8,0) \mathrm{BN}$ nanotubes were performed in [36]. The adsorption of $\mathrm{H}_{2}$ in structures found to be endothermic with respect to dissociation, with the small-diameter nanotube possessing the smaller barrier. Although chemisorption along the tube axis is energetically preferred, the barrier for dissociation is lower for chemisorption across the tube axis. This implies that chemisorbed hydrogen can be kinetically trapped in a higher energy state. Dopants that maximize the localization of the higher-occupied-molecular-orbital (HOMO) and lower-unoccupied-molecular-orbital (LUMO) states maximize hydrogen binding energies. C-dopants do not enhance $\mathrm{H}_{2}$ binding, whereas Si-dopants substituting for $\mathrm{N}$ provide $\mathrm{H}_{2}$ binding energies of $0.8 \mathrm{eV}$, at the upper end of the range required for hydrogen storage. The formation energy of most defects is reduced with increasing curvature except for the C-substitutionals. Vacancies do not reduce the barriers for $\mathrm{H}_{2}$ dissociation for strongly curved nanotubes. The surface stress induced by the nanotube curvature boosts the hydrogen storage capabilities of vacancies with the nitrogen vacancy chemisorbing $4 \mathrm{H}$ and allowing a $\mathrm{H}_{2}$ molecule to enter the interior of the tube. The hydrogen binding properties of $\mathrm{BN}$ systems strongly depend on existing defects and dopants. Pretreating of these systems so as to partially remove nitrogen should enhance $\mathrm{H}_{2}$ adsorption properties. The hydrogen absorption capacity of Ti-covered single-walled BN nanotube was investigated using first principles plane-wave (PW) method [37]. The weak interaction of $\mathrm{H}_{2}$ molecules with the outer surface of bare nanotube can be significantly enhanced upon functionalization by $\mathrm{Ti}$ atoms: each Ti atom adsorbed on tube can bind up to four $\mathrm{H}_{2}$ molecules with average binding energy suitable for room temperature storage.

The morphology of BN nanotubes with a collapsed structure has been discovered by a metal-catalyzed treatment [38]. The collapse causes the dramatic enlargement of a specific surface area of BN nanotubes and remarkably enhances the hydrogen storage capacity of BN nanotubes.

It was reported [39] that proteins are immobilized on boron nitride nanotubes. There is a natural affinity of a protein to $\mathrm{BN}$ nanotube: it can be immobilized on tube directly, without using of an additional coupling reagent. Besides, boron nitride nanotubes may be dissolved in organic solvents by wrapping them with a polymer [40]. 
It was proposed [41] that $\mathrm{BN}$ polymers, having the structures similar to organic polymers, can serve as a cheap alternative to inorganic semiconductors in designing modern electronic devices. Some related potential innovations, including band gap tuning, were also demonstrated.

The observed giant Stark effect significantly reduces the band gap of BN nanotubes and thus greatly enhances their utility for nanoscale electronic, electromechanical, and optoelectronic applications [42]. In particular, this effect may be important for tuning the band gap of $\mathrm{BN}$ nanotubes for applications as a nanoscale field-effecttransistor (FET).

Boron nitride nanotubes have also manifested stable currents in field emission geometry and may be more stable than carbon nanotubes at high temperatures [43,44].

As it was mentioned, boron nitride nanotubes exhibit many similarities with the carbon ones (such as high Young modulus etc.) and might have superior unique mechanical, thermal, and electronic properties [45]. In addition, BN nanotubes are characterized by chemical inertness and poor wetting [46].

The factor that distinguishes $\mathrm{BN}$ from $\mathrm{C}$ is partial heteropolarity of the chemical bonding. For this reason, one more sphere of possible applications of BN nanotubes can be new pyroelectric and piezoelectric materials promising for applications in nanometer-scale sensors and actuators. The 3-fold symmetry of a BN sheet, the III-V analog to graphite, prohibits an electric polarization in its ground state. However, this symmetry is broken when the sheet is wrapped to form a BN tube. It was shown [47] that this leads to an electric polarization along the nanotube axis which is controlled by the quantum-mechanical boundary conditions of its electronic states around the tube circumference. Thus, the macroscopic dipole moment has an intrinsically nonlocal quantum-mechanical origin from the wrapped dimension. Combining first principles, tight-binding methods and analytical theory, the piezoelectricity of heteropolar (in particular, BN) nanotubes was found [48] to depend on their chirality and radius. This effect can be understood starting from the piezoelectric response of an isolated sheet along with a structure specific mapping from the sheet onto the tube surface. It was demonstrated that a linear coupling between the uniaxial and shear deformations occurs for chiral nanotubes, and the piezoelectricity of nanotubes is fundamentally different from its counterpart in a bulk material. First principles calculations of the spontaneous polarization and piezoelectric properties of BN nanotubes have shown [49] that they are excellent piezoelectric systems with response values larger than those of piezoelectric polymers. The intrinsic chiral symmetry of the nanotubes induces an exact cancellation of the total spontaneous polarization in ideal, isolated nanotubes of arbitrary indexes. But the breaking of this symmetry by the intertube interaction or elastic deformations induces spontaneous polarization comparable to that of wurtzite bulk semiconductors [50].

Multielement nanotubes comprising multiple SiC-core, an amorphous $\mathrm{SiO}_{2}$-intermediate layer, and outer shells made of $\mathrm{BN}$ and $\mathrm{C}$ layers separated in the radial direction with diameters of a few tens of $\mathrm{nm}$ and lengths up to 50 $\mu \mathrm{m}$ were synthesized by means of reactive laser ablation [51]. They resemble a coaxial nanocable with a semiconductor-insulator-semiconductor (SIS) geometry and suggest applications in nanoscale electronic devices that take advantage of this self-organization mechanism for multielement nanotube formation.

A theoretical description of electron irradiation of single-walled BN nanotubes was presented in [52]. As a first step, the anisotropy of the atomic emission energy threshold was obtained within extended molecular-dynamical (MD) simulations based on the density-functionaltheory (DFT) tight-binding method. As a second step, total cross section for different emission sites as a function of the incident electron energy was numerically derived. Two regimes were then described: at low irradiation energies (below $300 \mathrm{keV}$ ), atoms are ejected mostly from the upper and lower parts of the tube while at high energies (above $300 \mathrm{keV}$ ) atoms are ejected mostly from the side walls. Typical values of the total cross section of knock-on processes are obtained to vary from a fraction of barn (at side wall for $150 \mathrm{keV}$ electrons) up to around 20 barns (for $1 \mathrm{MeV}$ electrons). In $\mathrm{BN}$ nanotubes, the emission energy threshold maps were reported to show B sputtering to be more favorable for low irradiation energies, while $\mathrm{N}$ sputtering is more favorable at high energies. These calculations of the total knock-on cross section for nanotubes can be used as a guideline for TEM experimentalists using high energy focused beams to shape nanotubes, and also, more generally, if electron irradiation is used to change nanotube properties such as their optical behavior or conductivity.

Such wide field of possible technical and technological applications of boron nitride nanotubes makes useful theoretical research determining their main physical characteristics. In particular, for purposeful design of some materials and devices based on nanotubular BN, like the fibrous composites, tubular heterojunctions, other nanoelectronic devices, nanoreservoirs for hydrogen storage etc, it is very important to be able to predict reliably values of the ground-state parameters, especially, the molar binding energies and sizes of the nanotubes with given indexes and their relative stability. In present work, this task is solved for the most stable - achiral (zigzag and armchair) - single-walled forms. 
Paper is organized as follows. In Section 1, we have introduced methods of synthesis of the boron nitride nanotubes and their technological applications. Section 2 is a brief summary of the structural and binding data available on nanotubular boron nitride. In Section 3, the theoretical approach based on the quasi-classical approximation to the binding energy calculation and geometries is presented. In Section 4, results of the performed calculations are presented in the form of curves "molar binding energy - structural parameter". Section 5 is devoted to estimation of the nanotube lattice zero-point vibration energy. And finally, Section 6 discusses relative stability of the boron nitride nanotubes of various radii and makes an attempt to generalize the obtained results.

\section{Structural and Binding Data}

Let us start with a brief overview of the structural and binding data available on a boron nitride diatomic molecule, isolated sheet, and nanotubular form.

\subsection{Molecular Boron Nitride}

The diatomic molecule BN can be considered as a simplest (degenerated) form for boron nitride nanotubes. In general, electronic theory of substance considers a diatomic molecule as a special problem for its intermediate structural and, consequently, electronic properties between mono- and polyatomic systems. Peculiarities are related mainly with the system axial symmetry and uniqueness of the structural parameter - interatomic distance $d$. Unlike the solid state or nanoscale boron nitrides, which are materials with a diversity of technical and industrial applications, BN molecule, which exists under the extreme conditions, is only of academic interest as a "building block" for two- and three-dimensional boron nitride structures. From the standard thermochemical data, the energy of $\mathrm{B}-\mathrm{N}$ bond at the equilibrium length is known to be considerably higher compared with those of $\mathrm{B}-\mathrm{B}$ and $\mathrm{N}-\mathrm{N}$ bonds. In addition, any stable regular $\mathrm{BN}$ structure is a network of atomic rings with alternating atoms such that the nearest-neighbor environment of both $\mathrm{B}$ and $\mathrm{N}$ atoms consists of only $\mathrm{B}-\mathrm{N}$ bonds. Therefore, the B-N bond length is a key interatomic distance in the analysis of boron-nitrogen binding.

There are known some old first principles and semiempirical investigations for boron-nitrogen interaction (see $[53,54])$. Applying a self-consistent-field (SCF) procedure to the $\mathrm{BN}$ molecule in [55], it was calculated molecular orbitals (MOs) in order to minimize total energy of the diatomic system. Then using the spectroscopic data available for the corresponding ground state, the $\mathrm{BN}$ molecule dissociation energy value $E$ was found to be $4.6 \mathrm{eV}$. According to the original theoretical approach of [56], the equilibrium interatomic distance in this molecule equals to $1.307 \AA$. At the same time, spectroscopic parameters characterizing the calculated boron-nitrogen interaction potential curve lead to the dissociation energy estimation of $5.05 \mathrm{eV}$. Nearly the same theoretical value for the bond length of $1.320 \AA$ was suggested in [57]. In [58], a short-ranged classical-forcefield (CFF) modeling of $\mathrm{BN}$ modifications was performed on the basis of experimental and first principles solid-state and diatomic-molecular data. In particular, assuming that CFF can be correctly determined by a sum of only two-body interaction terms, the $\mathrm{B}-\mathrm{N}$ potential energy had been expressed analytically via Morse potential, which gave $d \approx 1.32521 \AA$ and $E \approx 5.0007 \mathrm{eV}$. However, it was noted [25] that standard forms of the pair interatomic potentials, such as the Morse, Mee-Grüneisen, Buckingham, and other potentials, converge slowly and, therefore, a cutoff procedure should be used. But, in such a case a non-physical jump on the potential radial function can arise. In order to eliminate this problem, based on the embedded atom method, a new $\mathrm{B}-\mathrm{N}$ interatomic potential was designed which fulfills the conditions for smooth end: the potential function and its derivative (i.e., the interatomic force) vanish at the cutoff radius. The equilibrium bond length of $1.4457 \AA$ and binding energy of $4.00 \mathrm{eV}$ were found to reproduce correctly relative stabilities of the boron nitride layered structures.

We also suggested [59] a theoretical, namely, quasiclassical method of calculation of the dependence the B-N interatomic binding energy $E$ upon the bond length $d$. The constructed $E=E(d)$ curve was shown to be useful for estimations of BN crystalline structures cohesion parameters as well. This function reveals standard behavior characteristic for the central pair potentials. $E(0)=-\infty$, and $E(d) \equiv 0$ if $d$ is equal or greater than the sum of $\mathrm{B}$ and $\mathrm{N}$ quasi-classical atomic radii $r_{(\mathrm{B})} \approx 2.30$ and $r_{(\mathrm{N})} \approx 1.70 \AA$, i.e., $d \geq r_{(\mathrm{B})}+r_{(\mathrm{N})} \approx 4.00$ $\AA$ (note that quasi-classical $\mathrm{B}-\mathrm{N}$ interatomic potential automatically fulfills the conditions for the smooth end at $\left.d=r_{(\mathrm{B})}+r_{(\mathrm{N})}\right)$, while within the intermediate region $0<d<r_{(\mathrm{B})}+r_{(\mathrm{N})}$ it is an oscillating function with several maxima. Among these maxima only one is available kinetically and, therefore, it corresponds to the equilibrium. Analysis of the piece of the quasi-classical $E=E(d)$ curve for BN diatomic molecule, in the vicinity of this maximum, yields the values of bond length of $1.55 \AA$ and binding energy of $4.51 \mathrm{eV}$. Same dependence determined earlier $[60,61]$ within the frames of another quasi-classical parameterization scheme (for this purpose the screening factor of the potential affecting the given electron in interacting atom was approximated by the 
radial polynomial, not by the constant) is relatively flat and leads to the estimations of $1.58 \AA$ and $4.79 \mathrm{eV}$.

Thus, the spread in theoretical and semiempirical values for BN molecule binding energy is $(4.0-5.5) \mathrm{eV}$, which overlaps with the recommended [62] experimental dissociation energy value of $(4.0 \pm 0.5) \mathrm{eV}$. The available first principles and semiempirical calculations and thermochemical experimental data lead to the binding energy values of about (4-7) eV per B-N bond for various, differently coordinated, BN modifications (for sheet and nanotubular structures see below). Such kind of estimations may be considered to be in qualitative agreement with the quasi-classically calculated B-N bond energy as the ground state energetic parameters are quite sensitive to the atomic coordination. For this reason, we focus our attention on the differences in the bond length values between $\mathrm{BN}$ molecular and crystalline phases.

The quasi-classical values for the isolated $\mathrm{B}-\mathrm{N}$ bond length and other relevant theoretical and semiempirical data, which lie over the range (1.307-1.580) $\AA$, are overestimated in comparison with $1.281 \AA$ measured in ${ }^{11} \mathrm{~B}^{14} \mathrm{~N}$ molecule [63]. An explanation may be that $\mathrm{BN}$ molecular spectra [64] verify triplet ground state, but at the same time reveal a low-lying singlet state with longer bond.

Quasi-classically calculated interatomic vibration energy in a $\mathrm{B}-\mathrm{N}$ diatomic system of $0.178 \mathrm{eV} / \mathrm{mole}$ (the corresponding vibration quantum equals to $1435 \mathrm{~cm}^{-1}$ ) was found by fitting the quasi-classical $\mathrm{B}-\mathrm{N}$ potential curve with parabola $[60,65]$. This value is in good agreement (accurate within $5 \%$ ) with the values experimentally obtained for a neutral BN molecule of 0.187 (1514.6) [63] and $0.188 \mathrm{eV} /$ mole $\left((1519.0 \pm 0.2) \mathrm{cm}^{-1}\right.$, from the absorption spectra Fourier analysis for laser-induced molecular fluorescence) [64].

According to the SCF theoretical method of [56], the ground state vibration energy in molecular $\mathrm{BN}$ estimated as $0.179 \mathrm{eV} /$ mole $\left(1446 \mathrm{~cm}^{-1}\right)$, which is almost the quasiclassical result.

In [57], it was suggested the higher theoretical value of 0.217 (1750), what is close with $0.216 \mathrm{eV} /$ mole $(1740$ $\mathrm{cm}^{-1}$ ) measured in ionized molecule $\mathrm{BN}^{+}[66]$.

Studies of more complex molecular clusters of B and $\mathrm{N}$ are also interesting to get deeper insight into the defect formation processes in boron nitride nanotubes. Hightemperature Knudsen cell mass spectrometry was used to study the equilibria involving the $\mathrm{B}_{2} \mathrm{~N}$ molecule [67]. The thermal functions necessary to evaluate the mass spectrometric equilibrium data had been calculated from available experimental and theoretical molecular parameters. In particular, in some $\mathrm{B}_{2} \mathrm{~N}$ formation reactions changes in enthalpy have been measured. Room temperature atomization and formation enthalpies were determined to be 10.84 and $5.71 \mathrm{eV}$, respectively. At the same time, first-principles calculations were performed to estimate the electronic parameters of $\mathrm{B}_{2} \mathrm{~N}$, such as ionization energy and electron affinity.

Mixed clusters of $\mathrm{B}$ and $\mathrm{N}$ atoms $-\mathrm{B}_{2} \mathrm{~N}, \mathrm{BN}_{2}, \mathrm{~B}_{3} \mathrm{~N}$, $\mathrm{B}_{4} \mathrm{~N}, \mathrm{~B}_{2} \mathrm{~N}_{2}$, and $\mathrm{B}_{3} \mathrm{~N}_{2}$ - can be produced by sputtering of a solid state $\mathrm{BN}$ [68]. Atom ordering in assumed linear species had been derived from measurements of the mass distribution of both the positive and the negative products from the fragmentation of the anionic clusters in a gas target. As for neutral configurations, they were calculated. A tendency was found that a structure with the highest number of $\mathrm{B}-\mathrm{N}$ bonds is most stable both in neutral and anionic species (an exception is the $\mathrm{BN}_{2}$ molecule). In contrast to this, the species with the highest number of adjacent same atoms (except for triatomic chains) had the largest electron affinity.

\subsection{Boron Nitride Sheet}

The facts that boron nitride layered crystals and nanotubes may be prepared suggest the necessity of analyzing the hypothetic isolated infinite hexagonal layer, i.e., the $\mathrm{BN}$ sheet. Corresponding two-dimensional BN crystal is represented as a planar layer composed of regular hexagons with vertexes alternately occupied by $\mathrm{B}$ and $\mathrm{N}$ atoms. Classification and discussion of the BN haeckelite sheet structures, consisting of not only hexagonal atomic rings but also other even-membered rings, one can find in [69].

For the first time, the truncated crystal approach in the form of two semiempirical (standard and extended iterative Hückel) methods was applied to a two-dimensional hexagonal boron nitride structure [70]. The bond length was found to be 1.48 or $1.50 \AA$. However, when semiempirical calculations were performed on a two-dimensional periodic small cluster of the h-BN layer the equilibrium $\mathrm{B}-\mathrm{N}$ distance was computed as $1.441 \AA$ [71]. In [72], the 3-coordinated $\mathrm{B}_{12} \mathrm{~N}_{12}$ network of 6-membered atomic rings was examined theoretically. Namely, the total energy calculated using Hartree-Fock (HF) approach and DFT in local and gradient-corrected forms was minimized with respect to the $\mathrm{B}-\mathrm{N}$ bond length. But, "graphitic" isomer $\mathrm{B}_{12} \mathrm{~N}_{12}$ is only a fragment of the BN sheet and its geometry appears to be somewhat distorted because of finite sizes. As is to be expected, slight deviations of the bonds' angles from the ideal value of $120^{\circ}$ were observed for the bonds of atoms forming the central hexagon: $-(2.52-2.65)^{\circ}$ for $\mathrm{B}$ atoms and $+(2.52-2.65)^{\circ}$ for $\mathrm{N}$ atoms. There were also obtained number of unequal bond lengths: (1.266-1.283), (1.371-1.378), (1.427-1.442), (1.434-1.444), (1.520-1.536), and (1.553-1.576) A. The finiteness of the quasi-classical atomic radii allowed us to obtain the $\mathrm{B}-\mathrm{N}$ bond length for an infinite boron nitride sheet within the initial quasi-classical approxima- 
tion [73]. The calculated dependence of the molar binding energy on the lattice constant exhibits a maximum of $23.0 \mathrm{eV}$ at $2.64 \AA$, which should correspond to the equilibrium state for an isolated hexagonal layer (analytical optimization [74] of the lattice parameter using the binding energy calculated in quasi-classical approximation, which is possible only by neglecting the vibration energy, yields slightly different values: $23.2 \mathrm{eV}$ and $2.66 \AA$, respectively). The lattice constant of $2.64 \AA$ implies B-N bond length of $1.52 \AA$. Correction in energy introduced by zero-point vibrations was estimated as $0.242 \mathrm{eV} / \mathrm{mole}$ $[65,73]$.

The quasi-classical bond length of $1.52 \AA$ in an isolated $\mathrm{BN}$ sheet is in reasonable agreement (accurate within $4.6 \%$ ) with the bond length of $1.45 \AA$ observed in layers of real h-BN crystals. At first glance, the surprising thing is that the theoretical result for the isolated layer is in better agreement (with the accuracy of (2.6-3.8) $\%$ ) with the bond lengths in tetrahedrally coordinated modifications c-BN (1.57 $\AA$ ) and w-BN (1.56 and 1.58 $\AA)$. However, it is worth noting that to a certain extent two-dimensional boron nitride looks like three-dimensional crystals c-BN and $\mathrm{w}-\mathrm{BN}$ : these three structures do not contain weak interlayer bonds, which occur in the h-BN layered modification. The lengths of (1.52-1.54) and (1.55-1.58) $\AA$ obtained in [72] for the bonds of atoms forming the central (almost undistorted) hexagon in $\mathrm{B}_{12} \mathrm{~N}_{12}$ plane-fragment are also in good agreement with the quasi-classical result found for an idealized infinite BN sheet. Another quasi-classical approach using a different scheme of parameterization, employed to calculate h-BN binding and zero-point vibration energies, slightly underestimates the intralayer bond length [75]. The plausible reason may be that the crystalline equilibrium configuration was selected to maximize its static binding energy with respect only to the layer lattice parameter, while the interlayer distance was fixed.

Summarizing other theoretical and semiempirical results concerning intralayer bond lengths in h-BN (and $\mathrm{r}-\mathrm{BN}$ ), one can state that all of them are in agreement with the experimental value of $1.446 \AA$ [76]. For instance, in [77] the total energy of h-BN crystal as a function of unit cell volume $V$ had been calculated using orthogonalized linear-combinations-of-atomic-orbitals (LCAOs) method within the local-density-approximation (LDA). The equilibrium was found at $V / V_{\text {exp }} \approx 0.998$, where $V_{\text {exp }}$ is the experimental value of $V$. Such result corresponds to the intralayer B-N distance of $1.438 \AA$. The calculations of [78] were also based on DFT within LDA, but PW expansion was used both for the pseudopotential (PP) and the wave-function. The computed total energies and, consequently, the intralayer bond lengths in h-BN and r-BN were nearly the same: 1.441 and $1.439 \AA$, respectively. The short-ranged CFF modeling of boron nitrides leads to exactly the same intralayer B-N bond lengths in both layered structures: $1.454 \AA$ [58]. The results presented show satisfactory accuracy for the quasiclassically determined boron-nitrogen binding characteristics: accuracies of quasi-classical approach to determine isolated $\mathrm{B}-\mathrm{N}$ bond length and length of bonds in solid state structure amount a few percents, 7.2 and $5.1 \%$, respectively. Thus, the quasi-classically obtained $\mathrm{B}-\mathrm{N}$ binding curve and its parameters mentioned above (namely, equilibrium bond length, binding energy, and vibration frequency) would be useful for investigations of compounds containing $\mathrm{B}-\mathrm{N}$ bonds and, especially, $\mathrm{BN}$ nanosystems.

As for the $\mathrm{BN}$ sheet binding and vibration energies, it is also reasonable to analyze correctness of the given predictions by comparing them with data available on the cohesion characteristics of h-BN layered crystals. As follows from standard thermochemical data, the binding energy of h-BN equals to $13.0 \mathrm{eV} / \mathrm{mole}$ [79]. The binding energies of $14.5,16.0$, and $14.4 \mathrm{eV} /$ mole were determined from semiempirical calculations performed using two variants of the semiempirical LCAOs method and an approach based on a periodic small-sized cluster [70,71]. Within the CFF potential model, the lower semiempirical estimate of $11.5 \mathrm{eV} / \mathrm{mole}$ was obtained [58]. In the framework of DFT, optimization of the structural parameters led to the theoretical binding energy of 12.5 $\mathrm{eV} / \mathrm{mole}$ [78]. Therefore, it can be expected that the molar binding energy for h-BN layered crystal lies in the range from 11.5 to $16.0 \mathrm{eV}$. The binding energy of 23.0 $\mathrm{eV} /$ mole found by the quasi-classical method for the isolated layer is considerably higher. However, when comparing these energies, one should take into account that interlayer bonds are substantially weaker than intralayer ones and that each atom in layered BN structures is involved in the formation of 5 bonds, of which only 3 are intralayer bonds. Consequently, if the interlayer energy is ignored as compared to the intralayer energy, we can assume that the binding energy per $\mathrm{B}-\mathrm{N}$ bond of similar modifications is equal to $3 / 5$ of the molar binding energy of the isolated layer. Making use of the result 23.0 $\mathrm{eV} /$ mole for layer, we find the molar binding energy of $3 / 5 \times 23.0 \mathrm{eV}=13.8 \mathrm{eV}$. Indeed, this energy is close to the midpoint $(13.75 \mathrm{eV})$ of the aforementioned energy range. On the other hand, the vibration energies of the isolated layer and layered crystals can be directly compared because the atoms of the low-dimensional system can execute vibrations in three independent directions in physical space. The quasi-classical result of 0.242 for two-dimensional BN agrees well with analogous calculations of 0.266 [65], with the semiempirical estimate of 0.225 for zero-point vibrations energy in h-BN [58], and 
coincides in order of magnitude with the estimate of 0.350 $\mathrm{eV} / \mathrm{mole}$ from the theoretical phonon spectrum [78].

A few words about the defects in BN layer. Using PP and expanded unit cell methods, it was found that Nvacancies in $\mathrm{BN}$ sheet, as well as di- and trivacancional clusters including neighbor defects in BN layer, are characterized by small binding energies [80]. Calculated spectra and oscillator strengths allow to interpret local bands of the optical absorption in pyrolitic h-BN crystals before and after irradiation by fast-neutrons, protons, and C-ions. By first-principles calculations the 20 structures $\mathrm{B}_{x} \mathrm{C}_{y} \mathrm{~N}_{z}$, derived from a hexagonal layer by placing $\mathrm{B}, \mathrm{N}$, or $\mathrm{C}$ atoms on each site, were considered [81] to investigate their relative stabilities. First-principles simulations of the interaction of molecular hydrogen $\mathrm{H}_{2}$ with the native and substitutional defects of a single hexagonal BN sheet were performed in [36]. The adsorption of $\mathrm{H}_{2}$ on structures found to be endothermic with respect to dissociation. Vacancies reduce the barriers for $\mathrm{H}_{2}$ dissociation.

The geometries of haeckelite BN sheets were constructed by DFT [82]. Their molar energy of cohesion is found to be higher (by $\sim 0.6 \mathrm{eV} / \mathrm{mole}$ ) than that of regular one.

\subsection{Nanotubular Boron Nitride}

The elementary form of a BN nanotube is a wrapped closed hexagonal surface inscribed in the cylinder. Such $\mathrm{BN}$ nanotubes can be found in regular - achiral, i.e., zigzag $(n, 0)$ or armchair $(n, n)$, and also in chiral $(n, m)$ forms, $0 \leq m \leq n$. Here $n$ and $m$ are the tube indexes. Their symmetry operators have been identified in [83]: each type belongs to different family of the non-symmorphic rod groups; armchair tubes with even $n$ are found to be centro-symmetric. The types and structures of the noncarbon, in particular, BN nanotubes were reviewed in [84]. In addition, the deformed regular or haeckelite nanotubes can exist. Concerning the haeckelite structures of BN tubes, a variety of chiral angles, including zigzag and armchair types, were observed. Depending on the structure formation kinetics characteristic for a given technology, BN nanotubes quite often take the bamboolike morphology, form of a nanoarch (i.e., half-tube at the ends closed by planes) etc. Real nanotubular structures are not infinite in length: they are definitely truncated.

The three main different possible morphologies of the cylindrical tube closing with flat [8], conical, and amorphous ends, as observed in experiments, were shown [85] to be directly related to the tube chirality. There are also possible rectangular BN nanotubes with linear defects on edges and with tips in the form of triangular flags. Such kinds of morphologies suggest the presence of energeti- cally unfavorable odd-membered atomic rings (i.e., pentagons and heptagons) in addition to favorable even-membered rings (e.g. squares).

As the growth of BN nanotubes cannot be directly observed and, consequently, the underlying microscopic mechanism is a controversial subject, in [86] first-principles MD simulation of the single-walled nanotube edges was performed. The behavior of growing $\mathrm{BN}$ nanotubes was found to strongly depend on the nanotube network chirality. In particular, open-ended zigzag tubes close rapidly into an amorphous tip, preventing further growth. In the case of armchair tubes, formation of squares traps the tip into a flat cap presenting a large central evenmembered ring. This structure is meta-stable and is able to revert to a growing hexagonal framework by incorporating incoming atoms. These findings are directly related to frustration effects, namely that $\mathrm{B}-\mathrm{N}$ bonds are energetically favored over $\mathrm{B}-\mathrm{B}$ and $\mathrm{N}-\mathrm{N}$ bonds.

The expressions of radii, $R_{(n, 0)}$ and $R_{(n, n)}$, of the zigzag and armchair, $(n, 0)$ and $(n, n), \mathrm{BN}$ nanotubes in terms of the index and the structure parameter $a$ were obtained [87]. The parameter $a$ corresponds to the lattice constant of the boron nitride layered crystals, i.e., intralayer B-B or N-N bonds lengths. Therefore, the B-N bond length $d$ equals to $a / \sqrt{3}$. The nanotube index $n=1,2,3, \ldots$ determines the number of atoms as nanotube unit cell consists of $2 n$ formula units BN. The estimations of radii of the single-walled BN nanotubes, for their part, can be used for predicting their most probable combinations in multi-walled structures.

Analyzing this problem, it is necessary to take into account that actually the question involves the average radii. A detailed study using the generalized tight-binding MD method has revealed [85] that, as a result of the dynamical relaxation, the structure acquires a wave-like or "rippled" surface in which B atoms are displaced inward, while $\mathrm{N}$ atoms are displaced outward. This relaxation is similar to the reconstruction occurring at clean surfaces of III-V type crystalline semiconductors. However a general feature of $\mathrm{BN}$ nanotubular systems is that stronger surface potentials are associated with regions of higher curvature [88]. Thus, the interlayer interaction in $\mathrm{BN}$ nanotubes differs from bonding in three-dimensional layered crystals. However, most probably, these distinctions for nanotubular BN are weak enough to change essentially the equilibrium interlayer distances which are observed in h-BN and r-BN crystals. This conclusion is also confirmed by the results of an experimental study of the multi-walled nanotubes by high-resolution electronic microscopy [89]. In these structures, like in three-dimensional layered BN crystals, hexagonal and rhombohedral stacking sequences can freely coexist in nanotube wallassembly. There are also possible some different cross- 
section flattening, as well as ordering of layers in nonspiral zigzag. According to first-principles total-energy calculations [90], the most favorable double-walled BN nanotubes are structures in which the inter-wall distances are about $3 \AA$, i.e., as interlayer distances in layered BN crystals. Therefore, due to the weakness of the interlayer van der Waals forces, various types of multi-walled BN nanotubes can exist. Consequently, it is more probable the formation of such multi-walled $\mathrm{BN}$ nanotubes in which the difference between the radii of adjacent regular nanotubes is close to the interlayer distance in a layered h-BN crystal, i.e., to half of the height of the hexagonal unit cell $c \approx 6.6612 \AA[91]$. Thus, the similarity between types of the adjacent nanotubes has no crucial importance.

In view of these factors, from the calculated singlewalled nanotubes [87] pairs most suitable for the formation of the stable double-walled BN nanotubes have been chosen. Remaining small divergences in sizes of the neighboring regular nanotubes can be compensated by defects and small chiral distortions. Such transformations of the zigzag and armchair nanotubes into chiral one will be accompanied, respectively, by the increase and decrease in their radii. If the difference in radius between regular nanotubes is more (less) than $c / 2$, the realization of structure in which the internal wall will be zigzag (armchair) and external - armchair (zigzag) is more probable. Hence, based on estimations of sizes of the single-walled BN nanotubes, it is possible to predict successfully the most stable double-walled forms. But, how can be solved the same problem for multi-walled nanotubes? A few words on the task. In this case, all over, it will be necessary to calculate radii of nanotubes with high indexes to choose sequences of single-walled nanotubes, whose radii are close to terms of arithmetic progression with common difference of $c / 2$. However, now only geometrical considerations will be insufficient. The point is that unlike double-walled nanotubes in multi-walled ones there are also medial layers. For this reason, the choice of the most stable multi-walled structure should be based on the comparison between the gains in energy, which are caused by the deviation from the equilibrium interlayer distance, on the one hand, and by chiral distortions, on the other hand.

We can mention some theoretical results available on binding properties and stabilities of BN nanotubes. Stabilities of the boron nitride nanotubular structures were studied by means of non-orthogonal tight-binding formalism [92]. The radii and energies of the BN nanotubes also were estimated by MD simulation [25] within the embedded atom model in which parameter $d$ took the experimental value $1.4457 \AA$ of the intralayer B-N bond length in real h-BN crystals. In [69], the binding energy of the regular BN nanotubes has been calculated within the DFT in generalized gradient approximation (GGA). Seeking equilibrium values of B-N bond length and radii, the geometry of the tubular 32-atom supercell was optimized. For $(8,0),(10,0)$ and $(4,4)$ tubes, it has been found $d \approx 1.46 \AA$, and for $(5,5)$ tube, $d \approx 1.45 \AA$. Within the frame of semiempirical calculations of the nanotubular piezoelectric characteristics performed by the method of modified neglecting of diatomic overlapping (MNDO) [50], their radii also were determined. In this case the dependence of energies on the bond length was calculated for the molecular fragments containing 3 or 4 elementary layers (presumably, in this work for $d$ the empirical value known for h-BN crystals was fixed as an equilibrium value).

The possible contribution of ionicity of bonds in boron nitride structures is important to explain the binding differences between $\mathrm{BN}$ tubes and similar $\mathrm{C}$ tubes [1]. In order to facilitate understanding and prediction of nanotube interactions in a multi-walled structure, the electrostatic potentials on both outer and inner surfaces of some single-walled BN nanotubes have been calculated at a HF Slater-type-orbital level [88]. Structures were optimized computationally. Fictitious hydrogen atoms were introduced at the ends of the open tubes to satisfy the unfulfilled valences. It was found that BN tubes have stronger and more variable surface potentials than graphitic ones. There are characteristic patterns of positive and negative sites on the outer lateral surfaces, while the inner ones are markedly positive.

The binding and vibrations in small-radius singlewalled BN nanotubes in [93] were studied by DFT using LDA. The results show that the chirality preference observed in experiments may be explained from the relative stability of the corresponding BN strips: the zigzag strips have larger binding energies and thus may be more easily formed. The smallest stable BN nanotube is found to be the $(5,0)$ zigzag nanotube. The dependence of the tube deformation energy on its radius $R$ was approximated by the formula $\Delta E[\mathrm{eV} / \mathrm{mole}]=5.82 / R^{2.09}[\AA]$. The phonon dispersions of $\mathrm{BN}$ nanotubes were calculated and the frequency of the radial breathing mode was found to be inversely proportional to the nanotube radius. The geometries of the BN nanotubes were also constructed in DFT [82]. Based on DFT calculations [69], it was found that the energies of haeckelite BN nanotubes exceed by $0.6 \mathrm{eV} / \mathrm{mole}$ those of corresponding hexagonal nanotubes. They are less stable in comparison with corresponding haeckelite sheets as well. However, still they are stable and can be synthesized. Energy of deformation (i.e., energy needed to wrap nanotube from its sheet prototype) for large haeckelite BN tubes extrapolated by the formula $\sim C / R^{\alpha}$, where $R$ is the tube radius, with different 
parameters $C$ and $\alpha \approx 2$ for different structures.

Using the symmetry properties in [83], it was determined the numbers of Raman- and infrared (IR)-active vibrations in single-walled BN nanotubes. In contrast to the regular carbon nanotubes, zigzag boron nitride tubes possess almost twice as many vibrations as armchair ones. An extensive first principles study of the phonons in BN nanotubes using perturbation DFT in the LDA was performed in [94], where, based on the non-symmorphic rod group symmetry of tubes, the Raman- and IR-active modes at the point of the one-dimensional Brillouin zone were evaluated. For zigzag and chiral nanotubes, the set of IR-active modes is a subset of the Raman-active modes. In particular, the radial breathing mode is not only Raman-, but also IR-active. However, for armchair tubes, the sets of IR- and Raman-active modes are disjoint. The frequencies of the active modes of zigzag, chiral, and armchair tubes were presented as a function of the tube diameter. They were compared with the frequencies obtained by the zone-folding method (i.e., by rolling of a BN sheet into a tube). Except for the highfrequency tangential modes, the zone-folding results are in very good agreement with the first principles calculations. The radial breathing mode frequency can be derived by folding a sheet of finite width. Finally, the effects of bundling on the phonon frequencies are shown to be small. First principles calculations of the nonresonant Raman spectra of zigzag and armchair BN nanotubes were presented in [95]. In comparison, a generalized bond-polarizability model, where the parameters are extracted from first principles calculations of the polarizability tensor of a $\mathrm{BN}$ sheet, was implemented. For light polarized parallel to the tube axis, the agreement between model and first principles spectra is almost perfect, but for perpendicular polarization, depolarization effects have to be included in the model in order to reproduce Raman intensities.

The possible dislocation dipoles as defect nuclei under tension in $\mathrm{BN}$ nanotubes were identified by dislocation theory and MD simulations [96]. Formation energies of the dipoles evaluated by gradient-corrected DFT are high and remain positive at large strains, thus suggesting great yield resistance of $\mathrm{BN}$ nanotubes. The dipole appears to be more favorable in spite of its homoelemental B-B and $\mathrm{N}-\mathrm{N}$ bonds. The resonant photoabsorption and vibration spectroscopy combined with scanning tunneling microscopy unambiguously identify the presence of Stone-Wales defects in BN nanotubes [97]. Based on extensive timedependent DFT calculations, it was proposed to resonantly photoexcite such defects in the IR and UV regimes as a means of their identification. Intrinsic defects in zigzag BN nanotubes, including single vacancy, divacancy, and Stone-Wales defects, were systematically investigated using DFT calculation in [98]. It was found that the structural configurations and formation energies of the topological defects are dependent on tube diameter. The results demonstrate that such properties are originated from the strong curvature effect in BN nanotubes. The scanning tunneling microscope images of intrinsic defects in the BN nanotubes also were predicted. The defected $\mathrm{BN}$ tubes with $\mathrm{C}$-substitutions were considered in [50].

The theoretical studies of the elastic properties of single-walled BN nanotubes, carried out using the total -energy non-orthogonal tight-binding parameterization, were reported in [99]. Tubes of different diameters, ranging from 0.5 to $2 \mathrm{~nm}$, were examined. The study found that in the limit of large diameters the mechanical properties of nanotubes approach those of the graphite-like sheet. The stiffness and plasticity of BN nanotubes was investigated [100] using generalized tightbinding MD and first principles total-energy methods. Due to the $\mathrm{B}-\mathrm{N}$ bond rotation effect, the compressed zigzag nanotubes were found to undergo anisotropic strain release followed by anisotropic plastic buckling. The strain is preferentially released toward $\mathrm{N}$ atoms in the rotated $\mathrm{B}-\mathrm{N}$ bonds. The tubes buckle anisotropically toward only one end when uniaxially compressed from both ends. Based on these results, a skin-effect-model of smart nanocomposite materials is proposed, which localizes the structural damage toward the surface side of the material. $\mathrm{B}-\mathrm{N}$ bond-rotation mode of plastic yield in $\mathrm{BN}$ nanotubes in [101] was investigated combining first principles computations with a probabilistic rate approach to predict the kinetic and thermodynamic strength. BN nanotubes yield defects have low activation, but high formation energies. In [50], elastic characteristics of BN nanotubes also were calculated applying MNDO method.

\section{Theoretical Basis}

Our calculations are based on the quasi-classical expression for binding molar energy of a substance, on the one hand, and on the geometric characterization of nanotubular boron nitride, on the other hand.

\subsection{Quasi-Classical Binding Energy of Substance}

Under the term 'substance' we imply polyatomic structures at the ground state, i.e., molecules, various clusters, and crystals. Consequently, any substance is considered as a non-relativistic electron system affected by the static external field of nuclei, which are fixed at the sites in structure, and the averaged SCF of electrons. Because of singularities at the points, where the nuclei are located, and electron shell effects as well the inner potential of 
substance does not satisfy the standard Wentzel-KramersBrillouin (WKB) quasi-classical condition of spatial smoothness. Nevertheless, beginning from Bohr's fundamental work 'On the constitution of atoms and molecules' up to the present the semi-classical analysis of the electronic spectrum has been widely used for light atoms and their small complexes. Besides, heavy atoms, large molecules, and crystals can be treated within the LDA using the total energy functional in the form of quasiclassical expansion. Success of quasi-classical approaches can be attributed to the diffuseness of atomic potentials. The expression for bounded electron states energies obtained by Maslov yields that precise and quasi-classical spectra are close to each other if the characteristic values of potential $\Phi_{0}$ and the radius of its action $R_{0}$ meet requirement $2 \Phi_{0} R_{0}^{2} \gg>1$ (here and below all relations are given in atomic units). For atomic potential $\Phi_{0} \sim Z / R$ and $R_{0} \sim R$ where $Z \geq 1$ is the atomic number and $R$ is the radius of electron cloud. Therefore, in case of atoms it is required that $2 Z R>1$. Even for light atoms their radii are several times larger than Bohr radius, $R>>1$. Thus atoms and all polyatomic structures indeed are quasi-classical electron systems and their structural and electronic characteristics can be calculated based on the quasi-classically parameterized electric charge density and electrical field potential distributions in atoms.

The values of $i$-th electron classical turning point radii $r_{i}^{\prime}$ and $r_{i}^{\prime \prime}, r_{i}^{\prime}<r_{i}^{\prime \prime}$, are obtained by solving the equations

$$
E_{i}=-\Phi_{i}(r)+\frac{l_{i}\left(l_{i}+1\right)}{2 r^{2}} \quad i=1,2,3, \ldots, Z,
$$

where $r$ denotes the distance from the center of atom, $\Phi_{i}(r)$ is the potential affecting the given electron, $E_{i}<0$ and $l_{i}$ are its energy and orbital quantum number, respectively.

In the ground state the inner classical turning point for relative motion of atomic nucleus and electron cloud coincides with the center of system. As for the corresponding outer classical turning point radius $\tilde{r}$, it is obtained by solving the equation

$$
\tilde{E}=Z \tilde{\Phi}(r),
$$

where $\tilde{E}<0$ denotes the energy associated with relative motion and

$$
\tilde{\Phi}(r)=-\frac{1}{Z-1}\left(\frac{Z^{2}}{r}-\sum_{i=1}^{i=Z} \Phi_{i}(r)\right)
$$

is the electron cloud potential affecting the nucleus.

In particular, using the quasi-classical parameterization based on the Coulomb-like atomic potentials $\Phi_{i}(r)$
$=Z_{i} / r$ we are able to get exact formulas

$$
\begin{gathered}
r_{i}^{\prime}=\frac{n_{i}\left(n_{i}-\sqrt{n_{i}^{2}-l_{i}\left(l_{i}+1\right)}\right)}{Z_{i}}, \\
r_{i}^{\prime \prime}=\frac{n_{i}\left(n_{i}+\sqrt{n_{i}^{2}-l_{i}\left(l_{i}+1\right)}\right)}{Z_{i}}, \\
\tilde{r}=\frac{2(Z-1)}{Z^{2}\left(Z^{2}-\sum_{i=1}^{i=Z} Z_{i}\right)^{2}}, \\
\tilde{E}=-\frac{Z^{3}\left(Z^{2}-\sum_{i=1}^{i=Z} Z_{i}\right)^{2}}{2(Z-1)^{2}} .
\end{gathered}
$$

Here $Z_{i}=n_{i} \sqrt{-2 E_{i}}$ is the effective charge of the screened nucleus and $n_{i}$ is the principal quantum number of $i$-th electron. The numerical values of $Z_{i}, \tilde{E}$, $r_{i}^{\prime}, r_{i}^{\prime \prime}$ and $\tilde{r}$ can be found by fitting the quasi-classical energy levels $E_{i}$ to ab initio (for instance HF) ones.

Quasi-classical limit implies the truncation of electron states charge densities outside the classical turning points and space-averaging within the range between them. In this case $i$-th electron partial charge density is approximated by the piecewise-constant radial function

$$
\begin{array}{ll}
\rho_{i}(r)=0 & r<r_{i}^{\prime} \\
=-\frac{3}{4 \pi\left(r_{i}^{\prime \prime 3}-r_{i}^{\prime 3}\right)} & r_{i}^{\prime}<r<r_{i}^{\prime \prime} . \\
=0 & r_{i}^{\prime \prime}<r
\end{array}
$$

As for the nucleus charge density, it should be averaged inside the $\tilde{r}$-sphere:

$$
\begin{array}{ll}
\tilde{\rho}(r)=\frac{3 Z}{4 \pi \tilde{r}^{3}} & 0<r<\tilde{r} . \\
=0 & \tilde{r}<r
\end{array} .
$$

Consequently, the full atomic charge density is expressed by the step-like radial function

$$
\begin{aligned}
& \rho(r)=\tilde{\rho}(r)+\sum_{i=1}^{i=Z} \rho_{i}(r) \equiv \rho_{k} \\
& r_{k-1}<r<r_{k} \quad k=1,2,3, \ldots, q,
\end{aligned}
$$

where $r_{k}$ and $\rho_{k}$ denote known constants which depend on parameters $r_{i}^{\prime}, r_{i}^{\prime \prime}$ and $\tilde{r}$,

$0 \equiv r_{0}<r_{1}<r_{2}<\cdots<r_{q}<\infty, q \leq 2 Z$ is the number of different homogenous-charge-density spherical layers in atom.

Using the Poisson equation, the radial dependence of the full atomic potential also can be approximated by the 
step-like function,

$$
\begin{gathered}
\varphi(r)=\frac{3 a_{k}\left(r_{k}^{2}-r_{k-1}^{2}\right)}{2\left(r_{k}^{3}-r_{k-1}^{3}\right)}+\frac{3 b_{k}\left(r_{k}^{5}-r_{k-1}^{5}\right)}{5\left(r_{k}^{3}-r_{k-1}^{3}\right)}+c_{k} \equiv \varphi_{k} \\
r_{k-1}<r<r_{k} \quad k=1,2,3, \ldots, q, \\
a_{k}=\sum_{i=1}^{i=k-1} \frac{4 \pi \rho_{i}\left(r_{i}^{3}-r_{i-1}^{3}\right)}{3}-\frac{4 \pi \rho_{k} r_{k-1}^{3}}{3}, \\
b_{k}=-\frac{2 \pi \rho_{k}}{3}, \\
c_{k}=\sum_{i=k+1}^{i=q} 2 \pi \rho_{i}\left(r_{i}^{2}-r_{i-1}^{2}\right)+2 \pi \rho_{k} r_{k}^{2},
\end{gathered}
$$

if it is substituted by the space-averaged values inside each of the $r_{k-1}<r<r_{k}$ intervals.

In the region $r>r_{q}$, both the charge density and potential vanish identically, $\rho(r) \equiv 0$ and $\varphi(r) \equiv 0$. Thus finite parameter $r_{q}$ acquires a meaning of the quasiclassical atomic radius.

Based on the presented step-like parameterization of the charge density and potential distributions in an atom, its quasi-classical total energy can be expressed in the following form:

$$
E_{(\text {Atom })}=\frac{\pi}{3} \sum_{k=1}^{i=q} \rho_{k} \varphi_{k}\left(r_{k}^{3}-r_{k-1}^{3}\right)<0 .
$$

Note that it includes the non-physical energy of selfaction, $E_{(\text {Atom }) \text { Self-Action }}>0$ which arises from substituting the charge density for the probability density. Its value can be easily calculated in the quasi-classical approximation and then excluded from the total energy.

When the molecular or crystalline charge densities and potentials are expressed by the superposition of the steplike atomic charge densities and potentials, respectively, the molar (i.e., per chemical formula unit of the substance) ground state static energy and its zero-point vibration correction are calculated as

$$
E_{\text {Static }}=\frac{1}{4} \sum_{\bar{t}} \sum_{(i)=1}^{(i)=N} \sum_{(k)=1}^{(k)=N} \sum_{j=1}^{j=q_{(i)}} \sum_{l=1}^{l=q_{(k)}} \rho_{(i) j} \varphi_{(k) l} V_{(i k) j l}\left(r_{(i k))}\right)<0,
$$

$$
E_{\text {Vibration }}=\frac{3}{2} \sum_{(i)=1}^{(i)=N} \sqrt{\sum_{\vec{t}}^{\prime} \sum_{(k)=1}^{(k)=N} \sum_{j=1}^{j=q_{(i)}} \sum_{l=1}^{l=q_{(k)}} \frac{\rho_{(i) j} \varphi_{(k) l}+\rho_{(k) l} \varphi_{(i) j}}{2 M_{(i)} r_{(i k) t}} \frac{\partial V_{(i k) j l}\left(r_{(i k))}\right)}{\partial r_{(i k) t}}}>0 .
$$

Here the primed summation symbol denotes the elimination of the terms with $\vec{t}=0$ and $i=k$; indexes in parentheses $(i)$ and $(k)$ denote the atoms in the molecule or crystal unit cell, $N$ is the full

number of atoms, $M_{(i)}$ is the mass of $i$-th atom, $\vec{t}$ is the crystal translational vector - in case of a molecule $\vec{t} \equiv 0, r_{(i k) \vec{t}}$ is a distance between atomic sites and

$$
V_{(i k) j l}\left(r_{(i k) \vec{t}}\right)=V\left(r_{(i) j}, r_{(k) l}, r_{(i k) \dot{t}}\right)+V\left(r_{(i) j-1}, r_{(k) l-1}, r_{(i k) \vec{t}}\right)-V\left(r_{(i) j}, r_{(k) l-1}, r_{(i k) \bar{t}}\right)-V\left(r_{(i) j-1}, r_{(k) l}, r_{(i k) \vec{t}}\right) \text {. }
$$

We have introduced an universal geometric function $V\left(R_{1}, R_{2}, D_{12}\right)$ which expresses the volume of the intersection of two spheres as a function of their radii $R_{1}$

$$
\begin{aligned}
& V\left(R_{1}, R_{2}, D_{12}\right)=\frac{4 \pi R_{1}^{3}}{3} \quad D_{12} \leq R_{2}-R_{1}, \\
& =\frac{4 \pi R_{2}^{3}}{3} \quad D_{12} \leq R_{1}-R_{2}, \\
& \begin{array}{c}
=\frac{\pi\left(R_{1}+R_{2}-D_{12}\right)^{2}\left(\left(R_{1}+R_{2}+D_{12}\right)^{2}-4\left(R_{1}^{2}-R_{1} R_{2}+R_{2}^{2}\right)\right)}{12 D_{12}} \\
=0 \quad R_{1}+R_{2} \leq D_{12} ; \\
\frac{\partial V\left(R_{1}, R_{2}, D_{12}\right)}{\partial D_{12}}=0 \quad D_{12} \leq R_{2}-R_{1},
\end{array} \\
& =0 \quad D_{12} \leq R_{1}-R_{2}, \\
& =-\frac{\pi\left(\left(R_{1}+R_{2}\right)^{2}-D_{12}^{2}\right)\left(D_{12}^{2}-\left(R_{1}-R_{2}\right)^{2}\right)}{4 D_{12}^{2}} \quad\left|R_{1}-R_{2}\right| \leq D_{12} \leq R_{1}+R_{2} \text {, } \\
& =0 \quad R_{1}+R_{2} \leq D_{12} . \\
& \left|R_{1}-R_{2}\right| \leq D_{12} \leq R_{1}+R_{2} \\
& E_{\text {Binding }}=\sum_{(i)=1}^{(i)=N}\left(E_{(i)}-E_{(i) \text { Self }- \text { Action }}\right) \\
& -\left(E_{\text {Static }}-E_{\text {Self-Action }}\right)-E_{\text {Vibration }}>0
\end{aligned}
$$
librium structure of substance is obtained by maximizing the molar binding energy of expected structures. 
with respect to their structural parameters.

However, neglecting the insignificant redistribution of valence electrons arisen from association of atoms into a molecular or crystalline structure, the quasi-classical selfaction energy of the substance is approximated by the sum of self-action energies of constituent atoms:

$$
E_{\text {Self-Action }} \approx \sum_{(i)=1}^{(i)=N} E_{(i) \text { Self-Aaction }} .
$$

And consequently, the binding energy approximately can be calculated without excluding of the self-action terms in advance:

$$
E_{\text {Binding }} \approx \sum_{(i)=1}^{(i)=N} E_{(i)}-E_{\text {Static }}-E_{\text {Vibration }}>0 .
$$

The expected errors of the quasi-classical approach can be estimated for the model inner potential in the form of the analytical solution of the Thomas-Fermi (TF) equation for the semi-classical atomic potential: structural and energy parameters of the electronic system determined within the initial quasi-classical approximation are shown to differ from their exact values by factors $(10 / 3 \pi)^{1 / 3} \approx 1.02 \sim 1$ and $(3 \pi / 10)^{2 / 3} \approx 0.96 \sim 1$, respectively. Thus the expected errors of the quasi-classical approach amount to a few percents. Even more, within the initial quasi-classical approximation there are no uncontrollable calculation errors due the finiteness of quasiclassical atomic radii - the pair interactions without series termination are truncated at the distances exceeded the sums of atomic radii.

A complete quasi-classical theory of substance including calculation schemes for structural and binding, as well as for electronic spectrum characteristics, one can find in $[102,103]$. These schemes have been applied successfully for $\mathrm{Na}$ molecular and crystalline structures [104], various diatomic molecules $[60,61]$, boron nanotubes $[105,106]$, and mainly for one-, two- and threedimensional structural modifications of boron nitride diatomic molecule, isolated plane sheet, hexagonal h-BN, cubic c-BN, and wurtzite-like w-BN crystals [59,60,64, 73-75,107,108].

\subsection{Geometries of the Boron Nitride Regular Single-Walled Nanotubes}

Summarizing previous subsection, one can conclude that equilibrium structural and binding parameters of the boron nitride nanotubes can be calculated quasi-classically based on analytical expressions describing their geometries. This task has been solved in $[87,108,109]$ for regular (achiral), i.e., zigzag $(n, 0)$ and armchair $(n, n) \mathrm{BN}$ nanotubes. A model of regular nanotubes used here assumes that all atomic sites are located on cylindrical surface at the vertexes of regular hexagons broken along
$\mathrm{B}-\mathrm{N}$ or $\mathrm{B}-\mathrm{B}$ and $\mathrm{N}-\mathrm{N}$ diagonals, i.e., the expected small differences in bond length distinguished by their orientation toward the tube axis are neglected.

Namely, radii $R_{(n, 0)}$ and $R_{(n, n)}$ of the zigzag and armchair nanotubes have been obtained $[87,108]$ in terms of the nanotube index $n=1,2,3, \ldots$ and the structure parameter $a$ :

$$
\begin{gathered}
R_{(n, 0)}=\frac{a}{4 \sin \pi / 2 n}, \\
R_{(n, n)}=\frac{\sqrt{5+4 \cos \pi / 2 n} a}{4 \sqrt{3} \sin \pi / 2 n} .
\end{gathered}
$$

As it was mentioned, the parameter $a$ corresponds to a lattice constant of the boron nitride layered crystals, i.e., to an intralayer $\mathrm{B}-\mathrm{B}$ or $\mathrm{N}-\mathrm{N}$ bond lengths. Therefore, the $\mathrm{B}-\mathrm{N}$ bond length $d$ equals to $a / \sqrt{3}$. The nanotube index $n$ determines the number of atoms, as a nanotube unit cell consists of $2 n$ formula units, $\mathrm{B}_{2 n} \mathrm{~N}_{2 n}$.

Detailed regular geometries of zigzag and armchair BN nanotubes have been described in [108,109] using cylindrical coordinates $(\rho, \varphi, z)$, which are useful for calculating binding energy.

A unit cell of zigzag nanotubes consists of 4 atomic rings in parallel planes perpendicular to the axis. There are 2 pairs of rings, each consisting of 2 planes with $n$ boron or $n$ nitrogen atoms. Obviously, the cylindrical coordinate $\rho$ for all atomic sites equals to the tube radius:

$$
\rho_{\mathrm{B}}=\rho_{\mathrm{N}}=R_{(n, 0)} .
$$

As for the coordinates $\varphi$ and $z$ in the first and second pairs of atomic rings, they equal to

$$
\begin{gathered}
\varphi_{\mathrm{B}}=\varphi_{\mathrm{N}}=2 l \pi / n, \\
z_{\mathrm{B}}=(6 m+1) a / 2 \sqrt{3}, \\
z_{\mathrm{N}}=(6 m-1) a / 2 \sqrt{3},
\end{gathered}
$$

and

$$
\begin{gathered}
\varphi_{\mathrm{B}}=\varphi_{\mathrm{N}}=(2 l+1) \pi / n, \\
z_{\mathrm{B}}=(3 m-1) a / \sqrt{3}, \\
z_{\mathrm{N}}=(3 m+1) a / \sqrt{3},
\end{gathered}
$$

respectively. Here $l=0,1,2, \ldots, n-1$ and $m=0, \pm 1, \pm 2, \ldots$ number atomic pairs in a given pair of the atomic rings and these rings themselves, respectively.

The unit cell of armchair nanotubes consists of 2 atomic rings in parallel planes perpendicular to the tube axis. For its part, each ring consists of $n$ boron and $n$ nitrogen atoms. The coordinate $\rho$ for all atomic sites again equals to the tube radius:

$$
\rho_{\mathrm{B}}=\rho_{\mathrm{N}}=R_{(n, n)}
$$


while the rest cylindrical coordinates in the first and second atomic rings equal to

$$
\begin{gathered}
\varphi_{\mathrm{B}}=\varphi_{1}+2 l \pi / n, \\
\varphi_{\mathrm{N}}=-\varphi_{1}+2 l \pi / n, \\
z_{\mathrm{B}}=z_{\mathrm{N}}=m a,
\end{gathered}
$$

and

$$
\begin{gathered}
\varphi_{\mathrm{B}}=-\varphi_{1}-2 \varphi_{2}+2 l \pi / n, \\
\varphi_{\mathrm{N}}=\varphi_{1}+2 \varphi_{2}+2 l \pi / n, \\
z_{\mathrm{B}}=z_{\mathrm{N}}=(2 m+1) a / 2,
\end{gathered}
$$

respectively. Here

$$
\begin{aligned}
& \sin \varphi_{1}=\frac{2 \sin \pi / 2 n}{\sqrt{5+4 \cos \pi / 2 n}}, \\
& \sin \varphi_{2}=\frac{\sin \pi / 2 n}{\sqrt{5+4 \cos \pi / 2 n}},
\end{aligned}
$$

and $l=0,1,2, \ldots, n-1$ and $m=0, \pm 1, \pm 2, \ldots$ number $\mathrm{B}$ or $\mathrm{N}$ atoms in atomic rings and these rings themselves.

Based on the above discussion, the distances between a given atomic site and the sites in the so-called central atomic pairs (with $l=m=0$ ) in zigzag and armchair nanotubes have been found.

For zigzag $\left(l=m=0: \varphi_{\mathrm{B}}=\varphi_{\mathrm{N}}=0, z_{\mathrm{B}}=a / 2 \sqrt{3}\right.$, and $\left.z_{\mathrm{N}}=-a / 2 \sqrt{3}\right)$ tubes

$$
\begin{aligned}
& \frac{\left({ }_{(n, 0)}^{l m} \mathrm{~B} 1-{ }_{(n, 0)}^{00} \mathrm{~B} 1\right)^{2}}{a^{2}}=\frac{\sin ^{2} l \pi / n}{4 \sin ^{2} \pi / 2 n}+3 m^{2}, \\
& \frac{\left({ }_{(n, 0)}^{l m} \mathrm{~B} 2-{ }_{(n, 0)}^{00} \mathrm{~B} 1\right)^{2}}{a^{2}}=\frac{\sin ^{2}(2 l+1) \pi / 2 n}{4 \sin ^{2} \pi / 2 n}+\frac{3(2 m-1)^{2}}{4}, \\
& \frac{{ }_{(n, 0)}^{\left.{ }^{m} \mathrm{~N} 1-{ }_{(n, 0)}^{00} \mathrm{~B} 1\right)^{2}}}{a^{2}}=\frac{\sin ^{2} l \pi / n}{4 \sin ^{2} \pi / 2 n}+\frac{(3 m-1)^{2}}{3}, \\
& \frac{\left({ }_{(n, 0)}^{m m} \mathrm{~N} 2-{ }_{(n, 0)}^{00} \mathrm{~B} 1\right)^{2}}{a^{2}}=\frac{\sin ^{2}(2 l+1) \pi / 2 n}{4 \sin ^{2} \pi / 2 n}+\frac{(6 m+1)^{2}}{12}, \\
& \frac{\left.{ }_{(n, 0)}^{l m} \mathrm{~B} 1-{ }_{(n, 0)}^{00} \mathrm{~N} 1\right)^{2}}{a^{2}}=\frac{\sin ^{2} l \pi / n}{4 \sin ^{2} \pi / 2 n}+\frac{(3 m+1)^{2}}{3}, \\
& \frac{\left({ }_{(n, 0)}^{l m} \mathrm{~B} 2-{ }_{(n, 0)}^{00} \mathrm{~N} 1\right)^{2}}{a^{2}}=\frac{\sin ^{2}(2 l+1) \pi / 2 n}{4 \sin ^{2} \pi / 2 n}+\frac{(6 m-1)^{2}}{12}, \\
& \frac{\left({ }_{(n, 0)}^{l m} \mathrm{~N} 1-{ }_{(n, 0)}^{00} \mathrm{~N} 1\right)^{2}}{a^{2}}=\frac{\sin ^{2} l \pi / n}{4 \sin ^{2} \pi / 2 n}+3 m^{2}, \\
& \frac{\left({ }_{(n, 0)}^{l m} \mathrm{~N} 2-{ }_{(n, 0)}^{00} \mathrm{~N} 1\right)^{2}}{a^{2}}=\frac{\sin ^{2}(2 l+1) \pi / 2 n}{4 \sin ^{2} \pi / 2 n}+\frac{3(2 m+1)^{2}}{4} .
\end{aligned}
$$

For armchair $\left(l=m=0: \varphi_{\mathrm{B}}=\varphi_{1}, \varphi_{\mathrm{N}}=-\varphi_{1}\right.$, and $\left.z_{\mathrm{B}}=z_{\mathrm{N}}=0\right)$ tubes,

$$
\frac{\left({ }_{(n, n)}^{l m} \mathrm{~B} 1-{ }_{(n, n)}^{00} \mathrm{~B} 1\right)^{2}}{a^{2}}=\frac{(5+4 \cos \pi / 2 n) \sin ^{2} l \pi / n}{12 \sin ^{2} \pi / 2 n}+m^{2},
$$

$$
\begin{aligned}
& \frac{\left({ }_{(n, n)}{ }^{l m} \mathrm{~B} 2-{ }_{(n, n)}^{00} \mathrm{~B} 1\right)^{2}}{a^{2}} \\
& =\frac{(5+4 \cos \pi / 2 n) \sin ^{2}(2 l-1) \pi / 2 n}{12 \sin ^{2} \pi / 2 n}+\frac{(2 m+1)^{2}}{4} \\
& \frac{\left({ }_{(n, n)}^{l m} \mathrm{~N} 1-{ }_{(n, n)}^{00} \mathrm{~B} 1\right)^{2}}{a^{2}} \\
& =\frac{(2 \sin (2 l-1) \pi / 2 n+\sin l \pi / n)^{2}}{12 \sin ^{2} \pi / 2 n}+m^{2}, \\
& \frac{\left({ }_{(n, n)}{ }^{l m} \mathrm{~N} 2-{ }_{(n, n)}^{00} \mathrm{~B} 1\right)^{2}}{a^{2}} \\
& =\frac{(\sin (2 l+1) \pi / 2 n+2 \sin l \pi / n)^{2}}{12 \sin ^{2} \pi / 2 n}+\frac{(2 m+1)^{2}}{4} \\
& \frac{\left({ }_{(n, n)}^{l m} \mathrm{~B} 1-{ }_{(n, n)}^{00} \mathrm{~N} 1\right)^{2}}{a^{2}} \\
& =\frac{(2 \sin (2 l+1) \pi / 2 n+\sin l \pi / n)^{2}}{12 \sin ^{2} \pi / 2 n}+m^{2} \\
& \frac{\left({ }_{(n, n)}{ }^{m} \mathrm{~B} 2-{ }_{(n, n)}^{00} \mathrm{~N} 1\right)^{2}}{a^{2}} \\
& =\frac{(\sin (2 l-1) \pi / 2 n+2 \sin l \pi / n)^{2}}{12 \sin ^{2} \pi / 2 n}+\frac{(2 m+1)^{2}}{4} \\
& \frac{\left({ }_{(n, n)}^{l m} \mathrm{~N} 1-{ }_{(n, n)}^{00} \mathrm{~N} 1\right)^{2}}{a^{2}}=\frac{(5+4 \cos \pi / 2 n) \sin ^{2} l \pi / n}{12 \sin ^{2} \pi / 2 n}+m^{2}, \\
& \frac{\left({ }_{(n, n)}{ }^{l m} \mathrm{~N} 2-{ }_{(n, n)}^{00} \mathrm{~N} 1\right)^{2}}{a^{2}} \\
& =\frac{(5+4 \cos \pi / 2 n) \sin ^{2}(2 l+1) \pi / 2 n}{12 \sin ^{2} \pi / 2 n}+\frac{(2 m+1)^{2}}{4}
\end{aligned}
$$

\section{Binding Energies in Dependence on Structural Parameter}

At first, based on the above stated relations and HF values of the atomic electron energy-levels tabulated in [110], the required quasi-classical parameters $r_{k}, \rho_{k}$, and $\varphi_{k}$ for constituent atoms B and $\mathrm{N}$ have been calculated. They are given in Tables $\mathbf{1}$ and $\mathbf{2}$, respectively.

Here the values are shown in atomic units with 7 significant digits in accordance with the accuracy of input data (HF energies). Such high accuracy is useful in interim calculations. As for the final results, they should be expressed in rounded figures with 3 or 4 significant digits (in $\AA$ or $\mathrm{eV}$ for structure or energy parameters, respectively) because it corresponds to the usual experimental errors when determining structure and energy parameters of a substance, and the relative errors of the semi-classical calculations aimed at finding theoretically these parameters for polyatomic systems amount to a few percents. 
Table 1. Quasi-classical parameters of step-like radial distributions of electron-charge-density and electric-field-potential in boron atom (in a.u.).

\begin{tabular}{cccccc}
\hline$k$ & 1 & 2 & 3 & 4 & 5 \\
\hline$r_{k}$ & $2.758476 \mathrm{E}-02$ & $5.098016 \mathrm{E}-01$ & $7.441219 \mathrm{E}-01$ & $4.021346 \mathrm{E}+00$ & $4.337060 \mathrm{E}+00$ \\
$\rho_{k}$ & $5.686514 \mathrm{E}+04$ & $-3.610951 \mathrm{E}+00$ & $-7.342212 \mathrm{E}-03$ & $-1.028341 \mathrm{E}-02$ & $-2.941197 \mathrm{E}-03$ \\
$\varphi_{k}$ & $2.105468 \mathrm{E}+02$ & $8.882329 \mathrm{E}+00$ & $3.652920 \mathrm{E}+00$ & $2.060720 \mathrm{E}-01$ & $6.135348 \mathrm{E}-04$ \\
\hline
\end{tabular}

Table 2. Quasi-classical parameters of step-like radial distributions of electron-charge-density and electric-field-potential in nitrogen atom (in a.u.).

\begin{tabular}{cccccc}
\hline$k$ & 1 & 2 & 3 & 4 & 5 \\
\hline$r_{k}$ & $9.446222 \mathrm{E}-03$ & $3.577244 \mathrm{E}-01$ & $5.498034 \mathrm{E}-01$ & $2.909074 \mathrm{E}+00$ & $3.204489 \mathrm{E}+00$ \\
$\rho_{k}$ & $1.982589 \mathrm{E}+06$ & $-1.044967 \mathrm{E}+01$ & $-1.939444 \mathrm{E}-02$ & $-4.126981 \mathrm{E}-02$ & $-2.187537 \mathrm{E}-02$ \\
$\varphi_{k}$ & $8.784581 \mathrm{E}+02$ & $2.022523 \mathrm{E}+01$ & $8.464698 \mathrm{E}+00$ & $5.096684 \mathrm{E}-01$ & $3.993358 \mathrm{E}-03$ \\
\hline
\end{tabular}

Using these parameters and expressions for the components of the quasi-classical molar binding energy and squared interatomic distances in zigzag and armchair BN nanotubes (Figures 1 and 2), their binding energies were calculated versus the structural parameter $a$ with spacing of 0.001 a.u., i.e., within the accuracy of 4 significant digits. The vibration energy is assumed to be zero when radicand in its formula becomes negative.

In order to carry out these massive calculations, a special computer code has been designed. Calculations were performed within the range of the structure parameter $a$ which varied from 1 to 13 a.u. with a step $\Delta a=0.001$ a.u. (that is quite enough to cover values having any physical sense). The nanotube indexes varied from $n=1$, covering zigzag and armchair nanotubes up to $(18,0)$ and $(10,10)$, respectively. The radii of the largest calculated species are approximately equal. They are sufficiently large for the tube molar binding energy to almost reach the "saturation" value, which is given by the binding energy of the planar hexagonal $\mathrm{BN}$ sheet. In order to make sure that such "saturation" indeed takes place, test species with very large indexes $(45,0)$ and $(26,26)$ (again with approximately equal radii) have also been calculated.

Figures 3-6 show $E_{\text {Binding }}(a)$ curves (and their trends near the peaks) for some zigzag and armchair BN nanotubes, respectively. One can see that at sufficiently small interatomic distances binding energy might take a large negative value that implies that the structure is unstable, while at sufficiently large interatomic distances the binding energy always equals to zero which reveals atomization of a structure. As for the intermediate distances, the molar binding energy is positive that is a signature of structural stability. In this case, general trend in binding energy value is decreasing. However, $E_{\text {Binding }}(a)$ curves are not monotonous, but with several extremes. Such kind of oscillatory behavior of the molar biding energy of

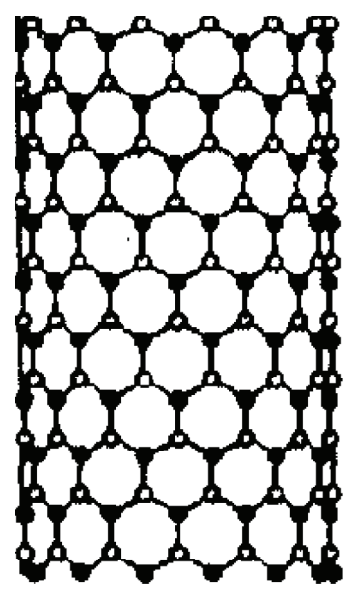

Figure 1. Structure of a zigzag BN nanotube.

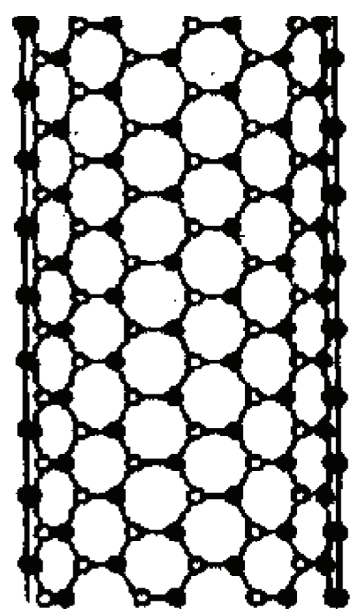

Figure 2. Structure of an armchair BN nanotube. 


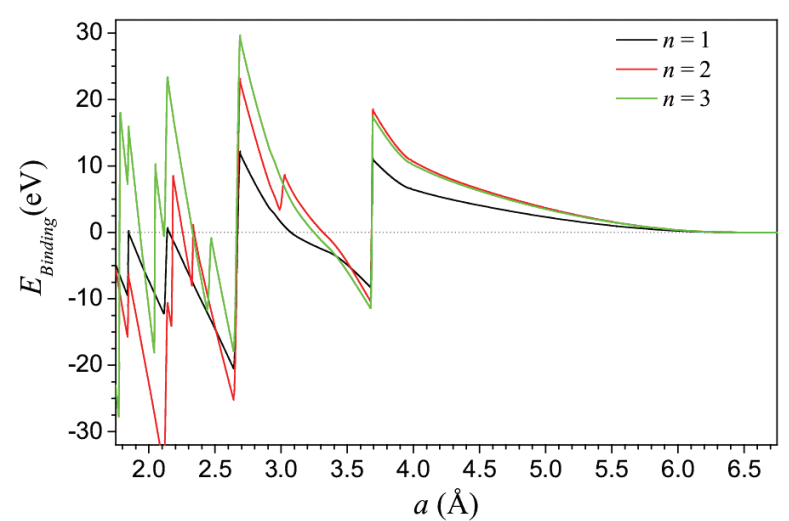

(a)

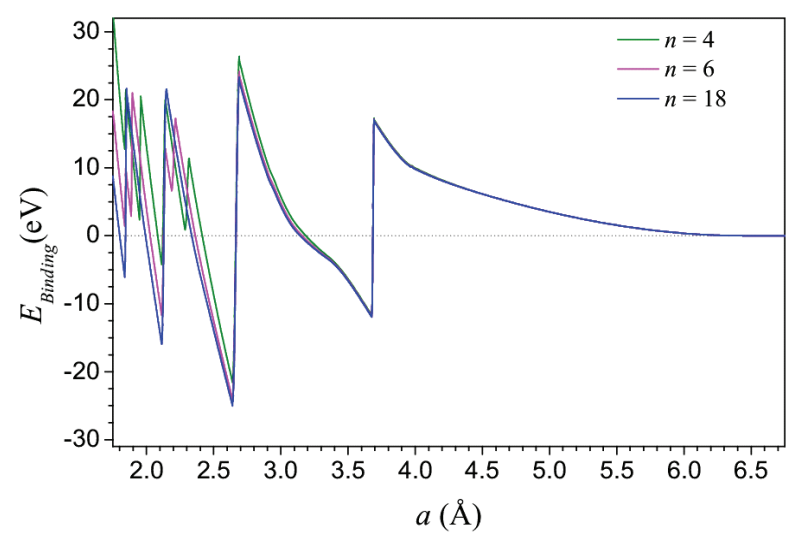

(b)

Figure 3. Molar binding energy of zigzag BN nanotubes vs. structural parameter $\boldsymbol{a}$ for different nanotube indexes $n$.

any atomic structure against the inter-atomic distances reflects electron-shell-structure of the constituent atoms (note that the interaction between particles of matter with forces non-monotonously decreasing with distance was foreseen as early as in 18th century by Boscovich [111], whose atomic theory was based only on abstract philosophical speculations).

Figures 7 and $\mathbf{8}$ show the binding energies for the two types of achiral nanotubes with the same index $n$ (namely $(3,0)$ and $(3,3)$ nanotubes).

Let us discuss which of the peaks in these figures correspond to the equilibrium structure. The first peak from the right is lower than the successive one. This second peak for all tubes located at $a=5.085$ a.u. (2.691 $\AA$ ) seems to correspond to the realized stable $\mathrm{BN}$ nanotubular structures (the detailed behavior of $E_{\text {Binding }}(a)$ curves in its vicinity is shown in Figures 4, 6 and 8). The next peak, even being higher than this, can not been reached kinetically in standard laboratory conditions because they correspond to lower interatomic distances and these two peaks are separated by very deep and sufficiently wide

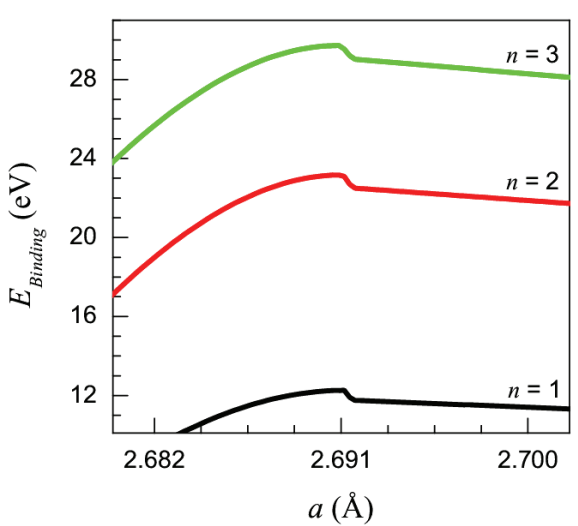

(a)

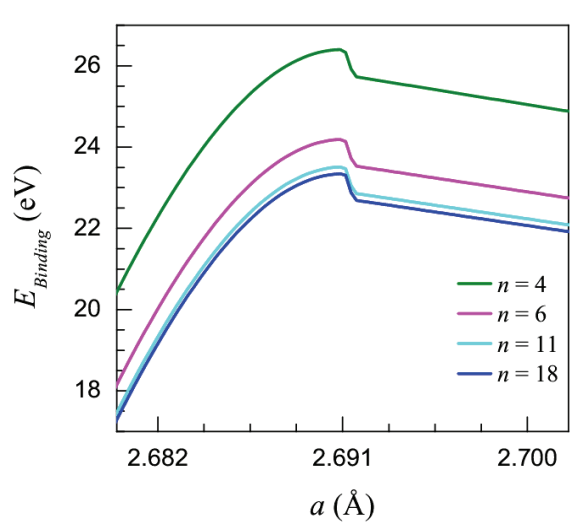

(b)

Figure 4. Molar binding energies of zigzag $B N$ nanotubes with different indexes $n$ near the peak $a=2.691 \AA$.

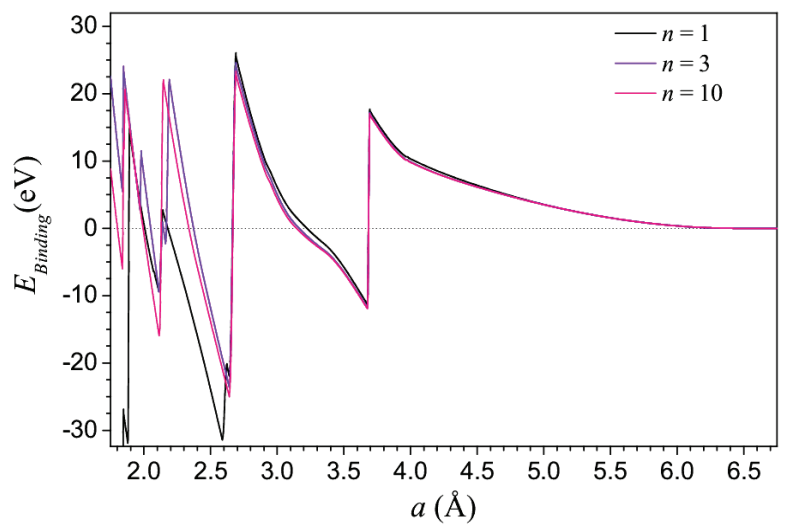

Figure 5. Molar binding energy of armchair BN nanotubes vs structural parameter $a$ for different nanotube indexes $n$.

minima, i.e., by high and wide potential barriers which can be overcome only at ultrahigh temperatures or tunneled only at ultrahigh pressures.

The obtained equilibrium binding energies of BN nanotubes of both achiral types are summarized in Table 3 . 


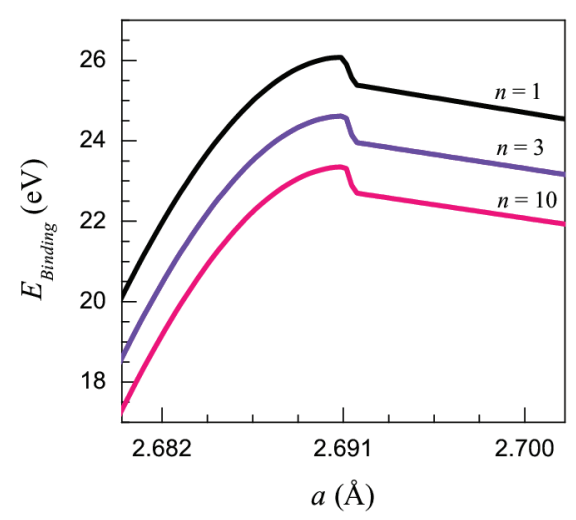

Figure 6. Molar binding energies of armchair BN nanotubes with different indexes $n$ near the peak $a=2.691 \AA$.

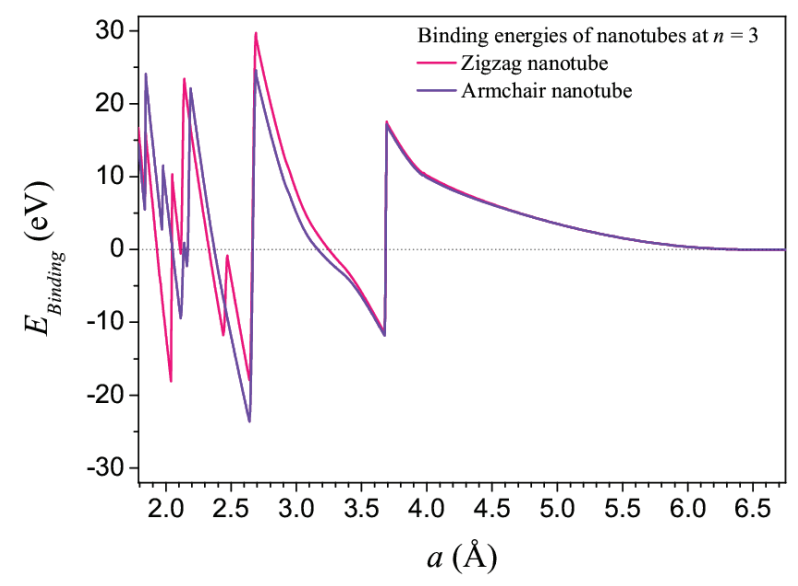

Figure 7. Dependence of molar binding energies of zigzag and armchair BN nanotubes with the nanotube index $n=3$ on the structural parameter $a$.

together with their radii calculated from formulas for equilibrium value of the structural parameter $a=2.691$ $\AA$.

Thus, the molar binding energies of small-sized nanotubes, both zigzag and armchair have peaks at $(3,0)$ and $(2,2)$, respectively. Then for large indexes the binding energies decrease toward the same constant value (see Figures 9 and 10).

Figure 11 presents the dependence of the molar binding energy of achiral $\mathrm{BN}$ nanotubes on their radii $R$. It reveals pairs of minima at $(1,0)$ and $(2,0)$, and maxima at $(1,1)$ and $(3,0)$, i.e., all the extremes are located in lowradii-region. At higher radii, the molar binding energy slowly decreases to the value of $23.26 \mathrm{eV}$, which, apparently, corresponds to that of the plane hexagonal $\mathrm{BN}$ sheet.

The obtained dependence $E_{\text {Binding }}(a)$ in its domain of monotonicity seems to be quite smooth. It allows us to extrapolate this curve also to chiral $\mathrm{BN}$ nanotubes (Figure 12) because the radius of a chiral tube $R_{(n, m)}$

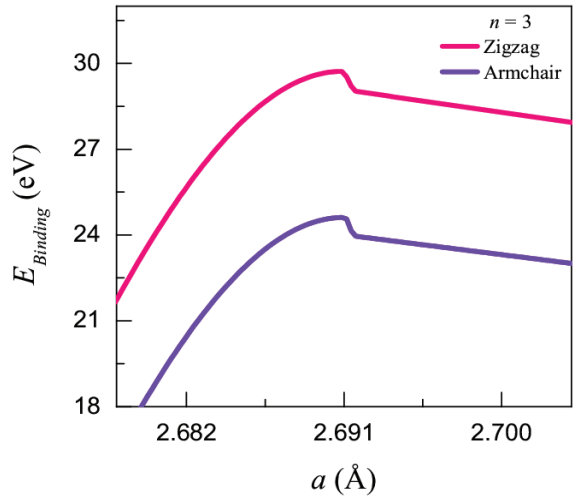

Figure 8. Molar binding energies of zigzag and armchair BN nanotubes with the index $n=3$ near the peak $a=2.691 \AA$.

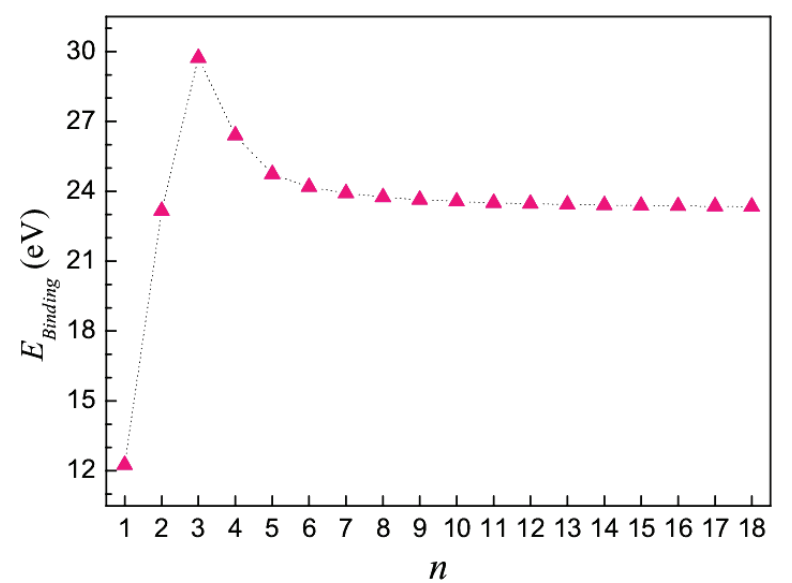

Figure 9. Molar binding energy of zigzag BN nanotubes for different nanotube indexes $n$ at the peak $a=2.691 \AA$.

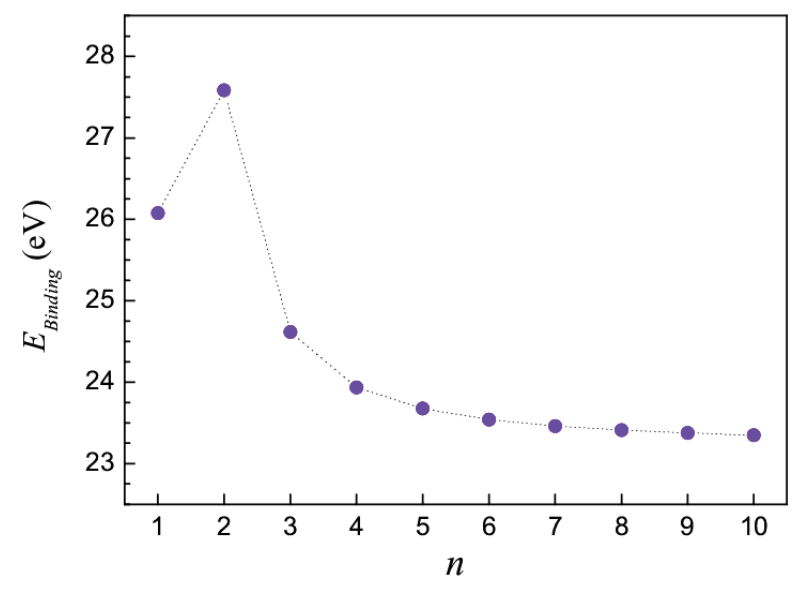

Figure 10. Molar binding energy of armchair BN nanotubes for different nanotube indexes $n$ at the peak $a=2.691 \AA$.

$(0 \leq m \leq n)$ and radii of the corresponding achiral tubes always meet the condition $R_{(n, 0)} \leq R_{(n, m)} \leq R_{(n, n)}$.

The results of the carried out calculations are pre- 
Table 3. Quasi-classically calculated radii and binding energies of BN nanotubes.

\begin{tabular}{|c|c|c|}
\hline Nanotube & Radius, $\AA$ & Binding Energy, eV/mole \\
\hline$(1,0)$ & 0.673 & 12.26 \\
\hline$(1,1)$ & 0.868 & 26.08 \\
\hline$(2,0)$ & 0.951 & 23.17 \\
\hline$(3,0)$ & 1.345 . & 29.72 \\
\hline$(2,2)$ & 1.537 & 27.59 \\
\hline$(4,0)$ & 1.758 & 26.40 \\
\hline$(5,0)$ & 2.177 & 24.74 \\
\hline$(3,3)$ & 2.260 & 24.62 \\
\hline$(6,0)$ & 2.599 & 24.19 \\
\hline$(4,4)$ & 2.993 & 23.94 \\
\hline$(7,0)$ & 3.023 & 23.92 \\
\hline$(8,0)$ & 3.448 & 23.76 \\
\hline$(5,5)$ & 3.729 & 23.68 \\
\hline$(9,0)$ & 3.874 & 23.64 \\
\hline$(10,0)$ & 4.300 & 23.57 \\
\hline$(6,6)$ & 4.668 & 23.54 \\
\hline$(11,0)$ & 4.727 & 23.51 \\
\hline$(12,0)$ & 5.154 & 23.47 \\
\hline$(7,7)$ & 5.207 & 23.46 \\
\hline$(13,0)$ & 5.581 & 23.43 \\
\hline$(8,8)$ & 5.947 & 23.41 \\
\hline$(14,0)$ & 6.008 & 23.41 \\
\hline$(15,0)$ & 6.436 & 23.39 \\
\hline$(9,9)$ & 6.687 & 23.37 \\
\hline$(16,0)$ & 6.863 & 23.37 \\
\hline$(17,0)$ & 7.291 & 23.35 \\
\hline$(10,10)$ & 7.428 & 23.35 \\
\hline$\ldots$ & $\ldots$ & $\ldots$ \\
\hline$(45,0)$ & 19.276 & 23.26 \\
\hline$(26,26)$ & 19.290 & 23.26 \\
\hline
\end{tabular}

sented in Figures 13 and $\mathbf{1 4}$ in the form of surface plots where the molar binding energy $E_{\text {Binding }}(a, n)$ of zigzag and armchair BN nanotubes is shown as a function of the structural parameter $a$ and the nanotube index $n$.

\section{Zero-Point Vibration Energies}

First, let us emphasize some features characteristic for the quasi-classical procedure of estimation of the zero-point vibration energies.

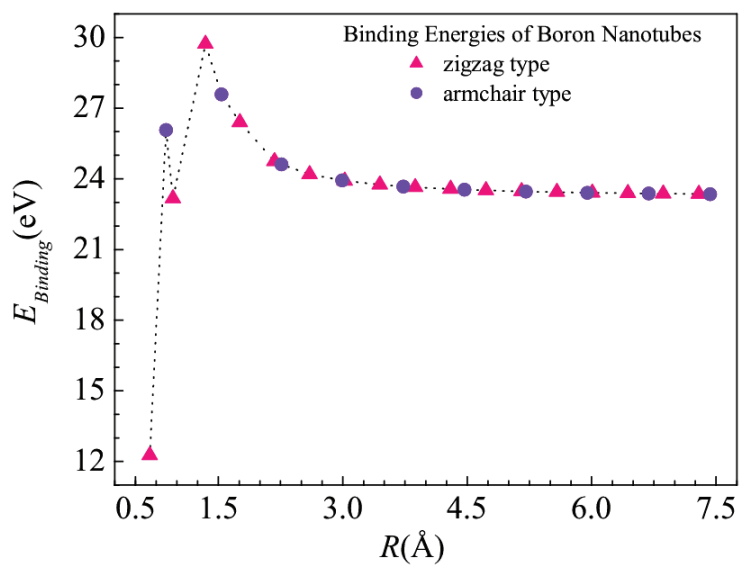

Figure 11. Molar binding energy of achiral BN nanotubes vs the nanotube radius $R$.

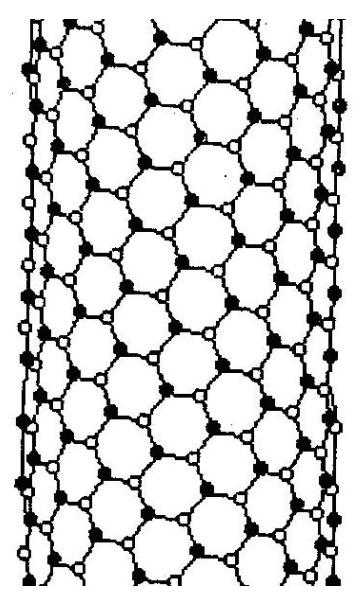

Figure 12. Structure of a chiral BN nanotube.

On the one hand, within the above formulated quasiclassical approach, all binding energy maxima are related with the onset of overlapping between certain regions of homogeneity of electric charge density and electric field potential in interacting atoms, constituents of the structure under the consideration. Namely, equilibrium point at $a \approx 5.085$ a.u. corresponds to the $\mathrm{B}-\mathrm{N}$ bond length of $d \approx 2.937$ a.u. which is a sum of radii $\mathrm{B} \quad r_{1} \approx 0.028$ a.u. and $\mathrm{N} \quad r_{4} \approx 2.909$ a.u. (Tables 1 and 2 ).

On the other hand, quasi-classical expression of the vibration energy is based on the parabolic approximation of the $E_{\text {Binding }}(a)$ curve and formula for the volume of the intersection of two spheres, $V\left(R_{1}, R_{2}, D_{12}\right)$, which is a continuously differentiable function of the inter-central distance $D_{12}$. However, one can see readily from expression of its first (continuous) derivative that second derivative is not a continuous function.

That is the reason why the parabolic approximation of the $E_{\text {Binding }}(a)$ curve in the immediate vicinity of the 


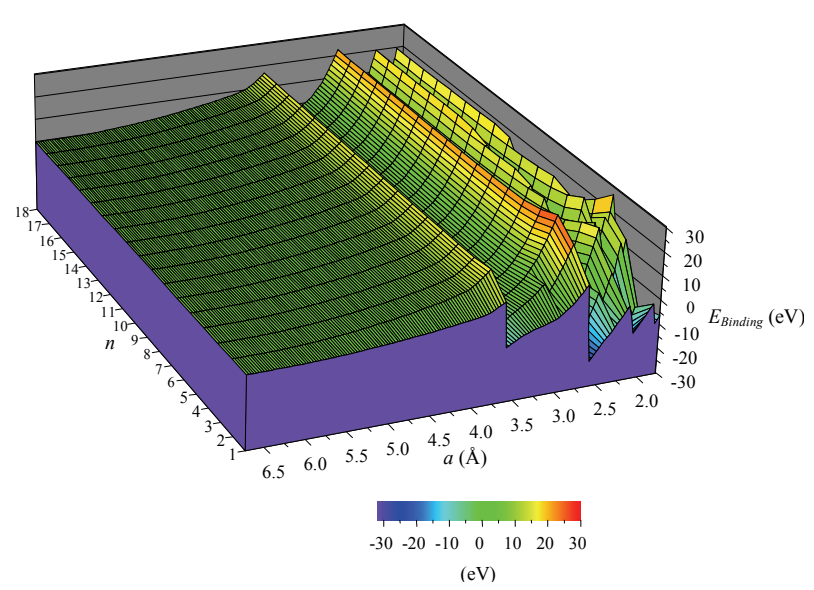

Figure 13. Surface plot of the molar binding energy $E_{\text {Binding }}(a, n)$ of a zigzag BN nanotube as a function of the structural parameter $a$ and nanotube index $n$.

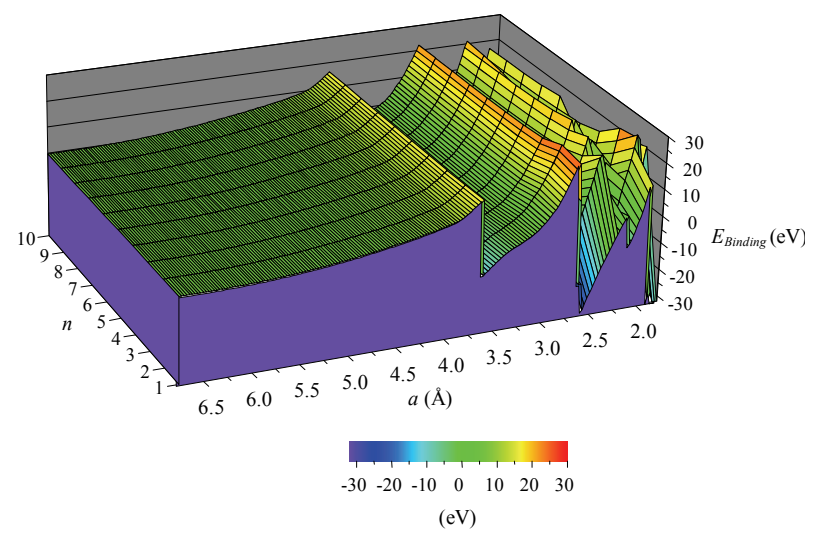

Figure 14. Surface plot of the molar binding energy $E_{\text {Binding }}(a, n)$ of an armchair BN nanotube as a function of the structural parameter $a$ and nanotube index $n$.

binding energy peak is impossible. According to formula, $E_{\text {Vibration }}$ is identically zero at the equilibrium point and is assumed to be identically zero also on the left in the vicinity of that point, where radicand in its formula becomes negative. As for the right side in the vicinity of the equilibrium point, the binding energy can be estimated for the nearest domain allowing parabolic approximation. Its half, i.e., arithmetic mean of the vibration energy left- and right-sided values can be considered as estimation for the vibration energy correction in the equilibrium. These values together with the correspondingly corrected binding energy are presented in Table 4.

The dependence of the molar vibration energy on the $\mathrm{BN}$ nanotube radius qualitatively reproduces that for the binding energy. However, this dependence is very weak and, thus, the molar vibration energy can be considered as almost independent from the tube radius, $\sim 0.3 \mathrm{eV} / \mathrm{mole}$.
Table 4. Quasi-classically calculated vibration energies of BN nanotubes.

\begin{tabular}{|c|c|c|}
\hline Nanotube & $\begin{array}{c}\text { Vibration Energy, } \\
\text { eV/mole }\end{array}$ & $\begin{array}{l}\text { Corrected Binding Energy, } \\
\mathrm{eV} / \mathrm{mole}\end{array}$ \\
\hline$(1,0)$ & 0.25 & 12.01 \\
\hline$(1,1)$ & 0.33 & 25.75 \\
\hline$(2,0)$ & 0.32 & 22.85 \\
\hline$(3,0)$ & 0.33 & 29.39 \\
\hline$(2,2)$ & 0.32 & 27.27 \\
\hline$(4,0)$ & 0.32 & 26.08 \\
\hline$(5,0)$ & 0.32 & 24.42 \\
\hline$(3,3)$ & 0.32 & 24.30 \\
\hline$(6,0)$ & 0.32 & 23.87 \\
\hline$(4,4)$ & 0.31 & 23.67 \\
\hline$(7,0)$ & 0.31 & 23.61 \\
\hline$(8,0)$ & 0.31 & 23.45 \\
\hline$(5,5)$ & 0.31 & 23.37 \\
\hline$(9,0)$ & 0.31 & 23.33 \\
\hline$(10,0)$ & 0.31 & 23.26 \\
\hline$(6,6)$ & 0.31 & 23.23 \\
\hline$(11,0)$ & 0.31 & 23.20 \\
\hline$(12,0)$ & 0.31 & 23.16 \\
\hline$(7,7)$ & 0.31 & 23.15 \\
\hline$(13,0)$ & 0.31 & 23.12 \\
\hline$(8,8)$ & 0.31 & 23.10 \\
\hline$(14,0)$ & 0.31 & 23.10 \\
\hline$(15,0)$ & 0.31 & 23.08 \\
\hline$(9,9)$ & 0.31 & 23.06 \\
\hline$(16,0)$ & 0.31 & 23.06 \\
\hline$(17,0)$ & 0.31 & 23.04 \\
\hline$(10,10)$ & 0.31 & 23.04 \\
\hline$\ldots$ & $\cdots$ & $\cdots$ \\
\hline$(45,0)$ & 0.31 & 22.95 \\
\hline$(26,26)$ & 0.31 & 22.95 \\
\hline
\end{tabular}

Of course, the vibration corrections to the binding energy are too weak to change character of the $E_{\text {Binding }}(R)$ dependence.

\section{Concluding Remarks}

The quasi-classically calculated structure parameter $a=$ $2.691 \AA$ of single-walled boron nitride nanotubes is in satisfactory agreement with experimental value for the h-BN layered crystals $a_{\text {exp }}=2.504 \AA[91]$, i.e., the diff- 
erence is about $7 \%$. As is mentioned above the overestimations in the structural parameter is characteristic for the quasi-classical approach. However, at least partially, this overestimation seems to be related with expansion of lattice of the single hexagonal layer (plane or cylindrical) if compared with that of the three-dimensional layered crystal.

It is also instructive to analyze the obtained spread of the molar zero-point vibration energy of $\mathrm{BN}$ nanotubes $(0.25-0.33) \mathrm{eV}$ and, in particular, its limit for ultra-largeradius tubes $0.31 \mathrm{eV}$ using the data available on the vibration characteristics of $\mathrm{h}-\mathrm{BN}$ layered crystal. The vibration energies of an isolated tubular layer and layered crystals can be directly compared as the atoms of the low-dimensional system can execute vibrations in three independent directions in physical space. Our quasi-classical estimations made for BN nanotubes agree well with analogous calculations (but in tight-binding approximation) for $\mathrm{BN}$ plane sheet of 0.27 , the semiempirical estimate of 0.23 for zero-point vibrations energy in $\mathrm{h}-\mathrm{BN}$, and the estimate of $0.35 \mathrm{eV} / \mathrm{mole}$ from the theoretical phonon spectrum (see Subsection 2.2).

We have found that the binding energies of $\mathrm{BN}$ single-walled nanotubes corrected with zero-point vibration energies lies within the interval (12.01-29.39) eV. In particular, the calculated corrected binding energy of the ultra-large-radius tube is predicted as $22.95 \mathrm{eV}$. Previous quasi-classical calculations (but in tight-binding approximation) performed for $\mathrm{BN}$ isolated plane sheet have given the binding energy $23.00 \mathrm{eV} / \mathrm{mole}$, which coincides in order of magnitude with this interval and agrees very well with present result obtained for large tubes. As it was demonstrated in Subsection 2.2, for its part the binding energy $\sim 23 \mathrm{eV} /$ mole for single-layer boron nitride structures should be in good agreement with binding energy data available for BN multi-layered structures. Summarizing the obtained results, it should be emphasized that a complex dependence of the BN nanotube molar binding energy on its radius is found out, though all the binding energy values are found to be positive, i.e., all tubes should be stable. However, they have rather different degrees of stability.

On the one hand, ultra-small-radius BN nanotubes $(1,0)$ and $(2,0)$ seem to be meta-stable, though their molar binding energies are positive, they are less than that for isolated hexagonal boron nitride layer. Especially the smallest $(1,0)$ tube structure degenerated in zigzag atomic strip should be meta-stable because its binding energy is only about half of this value. Such a structure can be realized only as an inner wall in a multi-walled tube. On the other hand, the formation probabilities for BN tubes with indexes $(1,1),(3,0)$, and $(4,0)$ should exceed that for isolated sheet. Among them the $(3,0)$ tube is well pronounced, formation of which is predicted to be energetically most preferable than the layer growth. Molar binding energies for other BN nanotubes slightly exceed that of sheet and their formation probabilities should be almost same as for layered crystal growth.

Finally, it should be noted that, in addition to the energy considerations concerning the relative stability of tubular structure, it is also necessary to take into account features characteristic to BN nanosystems, in view of the general equations derived for energy fluctuations of small completely open (incompressible) systems [112]. They show that the fluctuations should be unusually large because there are no constraints on the size of a system and, in addition, the fluctuations of the total or partial number of atoms in binary systems indirectly contribute to the fluctuations in their energy.

\section{Acknowledgements}

L. Chkhartishvili acknowledges the financial support from the Georgia National Science Foundation (GNSF) under the Project \# GNSF/ST 08/4-411-Geometry of the boron nitride nanostructures.

\section{REFERENCES}

[1] A. Rubio, J. L. Corkill and M. L. Cohen, "Theory of Graphitic Boron Nitride Nanotubes," Physical Review B, Vol. 49, No. 7, 1994, pp. 5081-5084.

[2] Z. Weng-Sieh, K. Cherrey, N. G. Chopra, X. Blasé, Y. Miyamoto, A. Rubio, M. L. Cohen, S. G. Louie, A. Zettl and R. Gronsky, "Synthesis of $\mathrm{B}_{x} \mathrm{C}_{y} \mathrm{~N}_{z}$ Nanotubules," Physical Review B, Vol. 51, No. 16, 1995, pp. 1122911232.

[3] N. G. Chopra, R. J. Luyken, K. Cherrey, V. H. Crespi, M. L. Cohen, S. G. Louie and A. Zettl, "Boron-Nitride Nanotubes," Science, Vol. 269, No. 5226, 1995, pp. 966-967.

[4] K. Suenaga, C. Colliex, N. Demoncy, A. Loieseau, H. Pascard and F. Willaime, "Synthesis of Nanoparticles and Nanotubes with Well-Separated Layers of Boron Nitride and Carbon," Science, Vol. 278, No. 5338, 1997, pp. 653655.

[5] K. Suenaga, F. Willaime, A. Loieseau and C. Colliex. "Organization of Carbon and Boron Nitride Layers in Mixed Nanoparticles and Nanotubes Synthesized by Arc Discharge," Applied Physics A, Vol. 68, No. 3, 1999, pp. 301-308.

[6] A. Loiseau, F. Willaime, N. Demoncy, G. Hug and H. Pascard, "Boron Nitride Nanotubes with Reduced Numbers of Layers Synthesized by Arc Discharge," Physical Review Letters, Vol. 76, No. 25, 1996, pp. 4737-4740.

[7] Y. Saito, M. Maida and T. Matsumoto, "Structures of Boron Nitride Nanotubes with Single-Layer and MultiLayers Produced by Arc Discharge," Japanese Journal of Applied Physics, Vol. 38, No. 1A, 1999, pp. 159-163.

[8] Y. Saito and M. Maida, "Square, Pentagon, and Heptagon 
rings at BN Nanotube Tips," Journal of Physical Chemistry A, Vol. 103, No. 10, 1999, pp. 1291-1293.

[9] D. Golberg, Y. Bando, M. Eremets, K. Takemura, K. Kurashima and H. Yusa "Nanotubes in Boron Nitride Laser Heated at High Pressure," Applied Physics Letters, Vol. 69, No. 14, 1996, pp. 2045-2047.

[10] R. Sen, B. C. Satishkumar, A. Govindaraj, K. R. Harikumar, G. Raina, J.-P. Zhang, A. K. Cheetham and C. N. R. Rao, "B-C-N, C-N and B-N Nanotubes Produced by the Pyrolysis of Precursor Molecules over Co Catalysts," Chemical Physics Letters, Vol. 287, No. 5-6, 1998, pp. 671-676.

[11] D. Golberg, Y. Bando, W. Han, K. Kurashima and T. Sato, "Single-Walled B-Doped Carbon, B/N-Doped Carbon, and BN Nanotubes Synthesized from Single-Walled Carbon Nanotubes through a Substitution Reaction," Chemical Physics Letters, Vol. 308, No. 3-4, 1999, pp. 337-342.

[12] D. Golberg, W. Han, Y. Bando, L. Bourgeois, K. Kurashima and T. Sato, "Fine Structure of Boron Nitride Nanotubes Produced from Carbon Nanotubes by a Substitution Reaction," Journal of Applied Physics, Vol. 86, No. 4, 1999, pp. 2364-2366.

[13] Y. Chen, L. T. Chadderton, J. F. Gerald and J. S. Williams, "A Solid-State Process for Formation of Boron Nitride Nanotubes," Applied Physics Letters, Vol. 74, No. 20, 1999, pp. 2960-2962.

[14] T. S. Bartnitskaya, G. S. Oleinik, V. V. Pokropivnyi, N. V. Danilenko, V. M. Vershchaka and A. V. Kotko, "Nanotubes from Graphite-Like BN," Superhard Materials, No. 6, 1998, pp. 71-74.

[15] T. S. Bartnitskaya, G. S. Oleinik, A. V. Pokropivnyi and V. V. Pokropivnyi, "Synthesis, Structure, and Formation Mechanism of Boron Nitride Nanotubes," JEPT Letters, Vol. 69, No. 2, 1999, pp. 163-168.

[16] C. Colazo-Davila, E. Bengu, L. D. Marks and M. Kirk, "Nucleation of Cubic Boron Nitride Thin Films," Diamond Related Materials, Vol. 8, No. 6, 1999, pp. 10911100.

[17] T. Oku, T. Hirano, M. Kuno, T. Kusunose, K. Niihara and K. Suganuma, "Synthesis, Atomic Structures, and Properties of Carbon and Boron Nitride Fullerene Materials," Materials Science and Engineering B, Vol. 74, No. 1-3, 2000, pp. 206-217.

[18] J. Wang, V. K. Kayastha, Y. K. Yap, Z. Fan, J. G. Lu, Z. Pan, I. N. Ivanov, A. A. Puretzky and D. B. Geohegan, "Low Temperature Growth of Boron Nitride Nanotubes on Substrates," Nano Letters, Vol. 5, No. 12, 2005, pp. 2528-2532.

[19] L. L. Sartinska, S. Barchikovski, N. Wagenda, B. M. Rud' and I. I. Timofeeva, "Laser Induced Modification of Surface Structures," Applied Surface Science, Vol. 253, No. 9, 2007, pp. 4295-4299.

[20] L. L. Sartinska, A. A. Frolov, A. Yu. Koval', N. A. Danilenko, I. I. Timofeeva and B. M. Rud', "Transformation of Fine-Grained Graphite-Like Boron Nitride Induced by Concentrated Light Energy," Materials Chem- istry and Physics, Vol. 109, No. 1, 2008, pp. 20-25.

[21] M. V. P. Altoe, J. P. Sprunck, J.-C. P. Gabriel and K. Bradley, "Nanococoon Seeds for BN Nanotube Growth," Journal of Materials Science, Vol. 38, No. 24, 2003, pp. 4805-4810.

[22] M. W. Smith, K. C. Jordan, C. Park, J.-W. Kim, P. T. Lillehei, R. Crooks and J. S. Harrison, "Very Long Single- and Few-Walled Boron Nitride Nanotubes via the Pressurized Vapor/Condenser Method," Nanotechnology, Vol. 20, No. 50, 2009, pp. 505604-505610.

[23] X. Blasé, J.-C. Charlier, A. de Vita and R. Car, "Theory of Composite $\mathrm{B}_{x} \mathrm{C}_{y} \mathrm{~N}_{z}$ Nanotube Heterojunctions," $A p$ plied Physics Letters, Vol. 70, No. 2, 1997, pp. 197-199.

[24] X. Blasé, J.-C. Charlier, A. de Vita and R. Car, "Structural and Electrical Properties of Composite $\mathrm{B}_{x} \mathrm{C}_{y} \mathrm{~N}_{z}$ Nanotubes and Heterojunctions," Applied Physics A, Vol. 68, No. 3, 1999, pp. 293-300.

[25] V. V. Pokropivnyj, V. V. Skorokhod, G. S. Oleinik, A. V. Kurdyumov, T. S. Bartnitskaya, A. V. Pokropivnyj, A. G. Sisonyuk and D. M. Sheichenko, "Boron Nitride Analogs of Fullerenes (the Fulborenes), Nanotubes, and Fullerites (the Fulborenites)," Journal of Solid State Chemistry, Vol. 154, No. 1, 2000, pp. 214-215.

[26] J. H. Lee, "A Study on a Boron-Nitride Nanotube as a Gigahertz Oscillator," The Journal of the Korean Physical Society, Vol. 49, No. 1, 2006, pp. 172-176.

[27] V. Verma and K. Dharamvir, "BNNT in Contact with h-BN Sheet and Other BNNT and DW-BNNT as GHz Oscillator," International Journal of Nanosystems, Vol. 1, No. 1, 2008, pp. 27-34.

[28] B. Baumeier, P. Krüger and J. Pollmann, "Structural, Elastic, and Electronic Properties of $\mathrm{SiC}, \mathrm{BN}$, and $\mathrm{BeO}$ Nanotubes," Physical Review B, Vol. 76, 2007, p. 085407.

[29] Y.-H. Kim, K. J. Chang and S. G. Louie, "Electronic Structure of Radially Deformed BN and $\mathrm{BC}_{3}$ Nanotubes," Physical Review B, Vol. 63, 2001, p. 205408.

[30] G. Y. Guo and J. C. Lin, "Second-Harmonic Generation and Linear Electro-Optical Coefficients of BN Nanotubes," Physical Review B, Vol. 72, 2005, p. 075416.

[31] G. Y. Guo and J. C. Lin, "Erratum: Second-Harmonic Generation and Linear Electro-Optical Coefficients of BN Nanotubes," Physical Review B, Vol. 77, 2008, p. 049901 (E).

[32] C. Zhi, Y. Bando, C. Tang and D. Golberg, "Engineering of Electronic Structure of Boron-Nitride Nanotubes by Covalent Functionalization," Physical Review B, Vol. 74, 2006, p. 153413.

[33] C. Zhi, Y. Bando, C. Tang and D. Golberg, "Publisher's Note: Engineering of Electronic Structure of Boron-Nitride Nanotubes by Covalent Functionalization," Physical Review B, Vol. 74, 2006, p. 199902 (E).

[34] W.-Q. Han, C. W. Chang and A. Zettl, "Encapsulation of One-Dimensional Potassium Halide Crystals within BN Nanotubes," Nano Letters, Vol. 4, No. 7, 2004, pp. 13551357.

[35] K. Yum and M.-F. Yu, "Measurement of Wetting Proper- 
ties of Individual Boron Nitride Nanotubes with the Wilhelmy Method Using a Nanotube-Based Force Sensor," Nano Letters, Vol. 6, No. 2, 2006, pp. 329-333.

[36] S. A. Shevlin and Z. X. Guo, "Hydrogen Sorption in Defective Hexagonal BN Sheets and BN Nanotubes," Physical Review B, Vol. 76, 2007, p. 024104.

[37] E. Durgun, Y.-R. Jang and S. Ciraci, "Hydrogen Storage Capacity of Ti-Doped Boron-Nitride and B/Be-Substituted Carbon Nanotubes," Physical Review B, Vol. 76, 2007, p. 073413 .

[38] C. Tang, Y. Bando, X. Ding, S. Qi and D. Golberg, "Catalyzed Collapse and Enhanced Hydrogen Storage of BN Nanotubes," Journal of the American Chemical Society, Vol. 124, No. 49, 2002, pp. 14550-14551.

[39] C. Zhi, Y. Bando, C. Tang and D. Golberg. "Immobilization of Proteins on Boron Nitride Nanotubes," Journal of the American Chemical Society, Vol. 127, No. 49, 2005, pp. 17144-17145.

[40] C. Zhi, Y. Bando, C. Tang, R. Xie, T. Sekiguchi and D. Golberg, "Perfectly Dissolved Boron Nitride Nanotubes Due to Polymer Wrapping," Journal of the American Chemical Society, Vol. 127, No. 46, 2005, pp. 1599615997.

[41] M. Côté, P. D. Haynes and C. Molteni, "Boron Nitride Polymers: Building Blocks for Organic Electronic Devices," Physical Review B, Vol. 63, 2001, p. 125207.

[42] M. Ishigami, J. D. Sau, Sh. Aloni, M. L. Cohen and A. Zettl, "Observation of the Giant Stark Effect in Boron-Nitride Nanotubes," Physical Review Letters, Vol. 94, 2005, p. 056804.

[43] J. Cumings and A. Zettl, "Mass-Production of Boron Nitride Double-Wall Nanotubes and Nanococones," Chemical Physics Letters, Vol. 316, No. 3-4, 2000, pp. 211-216.

[44] Y. Chen, J. Zou, S. J. Campbell and G. le Caer, "Boron Nitride Nanotubes: Pronounced Resistance to Oxidation," Applied Physics Letters, Vol. 84, No. 13, 2004, pp. 24302432.

[45] E. Bengu and L. D. Marks, "Single-Walled BN Nanostructures," Physical Review Letters, Vol. 86, No. 11, 2001, pp. 2385-2387.

[46] C. Tang, Y. Bando, Y. Huang, S. Yue, C. Gu, F. F. Xu and D. Golberg, "Fluorination and Electrical Conductivity of BN Nanotubes," Journal of the American Chemical Society, Vol. 127, No. 18, 2005, pp. 6552-6553.

[47] E. J. Mele and P. Král, "Electric Polarization of Heteropolar Nanotubes as a Geometric Phase," Physical Review Letters, Vol. 88, 2002, p. 056803.

[48] N. Sai and E. J. Mele, "Microscopic Theory for Nanotube Piezoelectricity," Physical Review B, Vol. 68, 2003, p. 241405.

[49] S. M. Nakhmanson, A. Calzolari, V. Meunier, J. Bernholc and M. Buongiorno Nardelli, "Spontaneous Polarization and Piezoelectricity in Boron Nitride Nanotubes," Physical Review B, Vol. 67, 2003, p. 235406.

[50] N. G. Lebedev and L. A. Chernozatonskiĭ, "Quantum-
Chemical Calculations of the Piezoelectric Characteristics of Boron Nitride and Carbon Nanotubes," Physics of the Solid State, Vol. 48, No. 10, 2006, pp. 2028-2034.

[51] Y. Zhang, K. Suenaga, C. Colliex and S. Iijima, "Coaxial Nanocable: Silicon Carbide and Silicon Oxide Sheathed with Boron Nitride and Carbon," Science, Vol. 281, No. 5379, 1998, pp. 973-975.

[52] A. Zobelli, A. Gloter, C. P. Ewels, G. Seifert and C. Colliex, "Electron Knock-on Cross Section of Carbon and Boron Nitride Nanotubes," Physical Review B, Vol. 75, 2007, p. 245402.

[53] I. V. Weiz, "Supplement II," In: K.-P. Huber and G. Herzberg, Constants of Diatomic Molecules, Part 2: Molecules $\mathrm{N}_{2}-\mathrm{ZrO}$, Mir, Moscow, 1984, pp. 295-366.

[54] O. I. Bukhtyarov, S. P. Kurlov and B. M. Lipenskikh, "Calculation of the Structure and Physicochemical Properties of B-Based Systems by the Computer Modeling Method," In: Abstracts of the 8th International Symposium on Boron, Borides, Carbides, Nitrides, and Related Compounds, Tbilisi, October 1984, pp. 135-136.

[55] J. L. Masse and M. Bärlocher, "Etude par la Méthode du Autocohérent (Self-Consistent) de la Molécule BN," Helvetica Chimia Acta, Vol. 47, No. 1, 1964, pp. 314318.

[56] Y. G. Khajt and V. I. Baranovskij, "Ab Initio Calculations of the BN Molecule," Journal of Structural Chemistry, Vol. 21, No. 1, 1980, pp. 153-154.

[57] H. Bredohl, J. Dubois, Y. Houbrechts and P. Nzohabonayo, "The Singlet Bands of BN," Journal of Physics B, Vol. 17, No. 1, 1984, pp. 95-98.

[58] C. M. Marian, M. Gastreich and J. D. Gale, "Empirical Two-Body Potential for Solid Silicon Nitride, Boron Nitride, and Borosilazane Modifications," Physical Review $B$, Vol. 62, No. 5, 2000, pp. 3117-3124.

[59] L. Chkhartishvili, "Quasi-Classical Analysis of BoronNitrogen Binding," In: Proceedings of the 2nd International Boron Symposium, Eskişehir, Turkey, September 2004, pp. 165-171.

[60] L. Chkhartishvili, D. Lezhava, O. Tsagareishvili and D. Gulua, "Ground State Parameters of $\mathrm{B}_{2}, \mathrm{BC}, \mathrm{BN}$, and BO Diatomic Molecules," Transactions of the AMIAG, Vol. 1, 1999, pp. 295-300.

[61] L. Chkhartishvili, D. Lezhava and O. Tsagareishvili, "Quasi-Classical Determination of Electronic Energies and Vibration Frequencies in Boron Compounds," Journal of Solid State Chemistry, Vol. 154, No. 1, 2000, pp. 148-152.

[62] A. Gaydon, "Dissociation Energies and Spectra of Diatomic Molecules," Chapman \& Hall, London, 1947.

[63] K. P. Huber and G. Herzberg, "Molecular Spectra and Molecular Structure: IV. Constants of Diatomic Molecules," van Nostrand Reinhold Co, New York, 1979.

[64] M. Lorenz, J. Agreiter, A. M. Smith and V. E. Bondybey, "Electronic Structure of Diatomic Boron Nitride," Journal of Chemical Physics, Vol. 104, No. 8, 1996, pp. 31433146 . 
[65] L. Chkhartishvili, "Zero-Point Vibration Energy within Quasi-Classical Approximation: Boron Nitrides," Proceedings of the TSU (Physics), Vol. 40, 2006, pp. 130-138.

[66] V. I. Vedeneev, L. V. Gurvich, V. N. Kondrat'ev, V. A. Medvedev and E. A. Frankevich, "Chemical Bonds Breaking Energies, Ionization Potentials and Electron Affinities," Academy of Sciences of USSR, Moscow, 1962.

[67] G. Meloni, M. Sai Baba and K. A. Gingerich, "Knudsen Cell Mass Spectrometric Investigation of the $\mathrm{B}_{2} \mathrm{~N}$ Molecule," Journal of Chemical Physics, Vol. 113, No. 20, 2000, pp. 8995-8999.

[68] R. Vanderbosch, "Gas-Phase Anions Containing B and N," Physical Review A, Vol. 67, 2003, p. 013203.

[69] S. V. Lisenkov, G. A. Vinogradov, T. Y. Astakhova and N. G. Lebedev, 'Non-Spiral 'Haeckelite-Type' BN-Nanotubes,” JETP Letters, Vol. 81, No. 7, 2005, pp. 431-436.

[70] A. Zunger, "A Molecular Calculation of Electronic Properties of Layered Crystals: I. Truncated Crystal Approach for Hexagonal Boron Nitride," Journal of Physics C, Vol. 7, No. 1, 1974, pp. 76-95.

[71] A. Zunger, "A Molecular Calculation of Electronic Properties of Layered Crystals: II. Periodic Small Cluster Calculation for Graphite and Boron Nitride," Journal of Physics C, Vol. 7, No. 1, 1974, pp. 96-106.

[72] D. L. Strout, "Structure and Stability of Boron Nitrides: Isomers of $\mathrm{B}_{12} \mathrm{~N}_{12}$," Journal of Physical Chemistry A, Vol. 104, No. 15, 2000, pp. 3364-3366.

[73] L. S. Chkhartishvili, "Quasi-Classical Estimates of the Lattice Constant and Band Gap of a Crystal: Two-Dimensional Boron Nitride," Physics of the Solid State, Vol. 46, No. 11, 2004, pp. 2126-2133.

[74] L. S. Chkhartishvili, "Analytical Optimization of the Lattice Parameter Using the Binding Energy Calculated in the Quasi-Classical Approximation," Physics of the Solid State, Vol. 48, No. 5, 2006, pp. 846-853.

[75] L. Chkhartishvili and D. Lezhava, "Zero-Point Vibration Effect on Crystal Binding Energy: Quasi-Classical Calculation for Layered Boron Nitride," Transactions of the GTU, Vol. 439, 2001, pp. 87-90.

[76] K.-H. Hellwege and O. von Madelung, Eds., "Boron Nitride (BN)," In: Landolt-Börnstein, Numerical Data and Functional Relationships in Science and Technology, New Series, Group III: Crystal and Solid State Physics, Volume 17: Semiconductors, Subvolume g: Physics of Non-Tetrahedrally Bonded Binary Compounds III, Springer-Verlag, Berlin, 1982.

[77] Y.-N. Xu and W. Y. Ching, "Calculation of Ground-State and Optical Properties of Boron Nitrides in the Hexagonal, Cubic, and Wurtzite Structures," Physical Review B, Vol. 44-I, No. 15, 1991, pp. 7784-7798.

[78] K. Albe, "Theoretical Study of Boron Nitride Modifications at Hydrostatic Pressures," Physical Review B, Vol. 55-II, No. 10, 1997, pp. 6203-6210.

[79] D. D. Wagmann, W. H. Evans, V. B. Parker, J. Halow, S. M. Bairly and R. H. Shumn, Eds., Selected Values of Chemical Thermodynamic Properties, National Bureau of
Standards, Washington, 1968.

[80] S. N. Grinyaev, F. V. Konusov and V. V. Lopatin, "Deep Levels of Nitrogen Vacancy Complexes in Graphite-Like Boron Nitride," Physics of the Solid State, Vol. 44, No. 2, 2002, pp. 286-293.

[81] M. S. C. Mazzoni, R. W. Nunes, S. Azevedo and H. Chacham, "Electronic Structure and Energetics of $\mathrm{B}_{x} \mathrm{C}_{y} \mathrm{~N}_{z}$ Layered Structures," Physical Review B, Vol. 73, 2006, p. 073108.

[82] S. V. Lisenkov, G. A. Vonogradov, T. Y. Astakhova and N. G. Lebedev, "Geometric Structure and Electronic Properties of Planar and Nanotubular BN Structures of the Haeckelite Type," Physics of the Solid State, Vol. 48, No. 1, 2006, pp. 192-198.

[83] O. E. Alon, "Symmetry Properties of Single-Walled Boron Nitride Nanotubes," Physical Review B, Vol. 64, 2001, p. 153408.

[84] V. V. Pokropivnyi, "Non-Carbon Nanotubes (Review). 2. Types and Structure," Powder Metallurgy and Metal Ceramics, Vol. 40, No. 11-12, 2002, pp. 582-594.

[85] M. Menon and D. Srivastava, "Structure of Boron Nitride Nanotubes: Tube Closing Versus Chirality," Chemical Physics Letters, Vol. 307, No. 5-6, 1999, pp. 407-412.

[86] J.-C. Charlier, X. Blase, A. de Vita and R. Car, "Microscopic Growth Mechanisms for Carbon and Boron-Nitride Nanotubes," Applied Physics A, Vol. 68, No. 3, 1999, pp. 267-273.

[87] L. S. Chkhartishvili, "On Sizes of Boron Nitride Nanotubes," In: Proceedings of the 18th International Symposium 'Thin Films in Optics in Nanoelectronics', Kharkiv, October 2006, pp. 367-373.

[88] Z. Peralta-Inga, P. Lane, J. S. Murray, S. Boyd, M. E. Grice, C. J. O'Connor and P. Politzer, "Characterization of Surface Electrostatic Potentials of Some $(5,5)$ and $(n, 1)$ Carbon and Boron/Nitrogen Model Nanotubes," Nano Letters, Vol. 3, No. 1, 2003, pp. 21-28.

[89] D. Golberg, Y. Bando, L. Bourgeois, K. Kurashima and T. Sato, "Insights into the Structure of BN Nanotubes," Applied Physics Letters, Vol. 77, No. 13, 2000, pp. 19791981.

[90] S. Okada, S. Saito and A. Oshiyama, "Inter-Wall Interaction and Electronic Structure of Double-Walled BN Nanotubes," Physical Review B, Vol. 65, No. 16, 2002, p. 165410.

[91] Y. B. Kuz'ma and N. F. Chaban, "Boron Containing Binary and Ternary Systems: Handbook," Metallurgiya, Moscow, 1990.

[92] E. Hernández, C. Goze, P. Bernier and A. Rubio, "Elastic properties of $\mathrm{C}$ and $\mathrm{B}_{x} \mathrm{C}_{y} \mathrm{~N}_{z}$ Composite Nanotubes," Physical Review Letters, Vol. 80, No. 20, 1998, pp. 45024505.

[93] H. J. Xiang, J. Yang, J. G. Hou and Q. Zhu, "First-Principles Study of Small-Radius Single-Walled BN Nanotubes," Physical Review B, Vol. 68, 2003, p. 035427.

[94] L. Wirtz, A. Rubio, R. A. de la Concha and A. Loiseau, "Ab initio Calculations of the Lattice Dynamics of Boron 
Nitride Nanotubes," Physical Review B, Vol. 68, 2003, p. 045425.

[95] L. Wirtz, M. Lazzeri, F. Mauri and A. Rubio, "Raman Spectra of BN Nanotubes: Ab Initio and Bond-Polarizability Model Calculations," Physical Review B, Vol. 71, 2005, p. 241402 (R).

[96] H. F. Bettinger, T. Dumitrică, G. E. Scuseria and B. I. Yakobson, "Mechanically Induced Defects and Strength of BN Nanotubes," Physical Review B, Vol. 65, 2002, p. 041406.

[97] Y. Miyamoto, A. Rubio, S. Berber, M. Yoon and D. Tománek, "Spectroscopic Characterization of Stone-Wales Defects in Nanotubes," Physical Review B, Vol. 69, 2004, p. 121413 (R).

[98] G. Y. Gou, B. C. Pan and L. Shi, "Theoretical Study of Size-Dependent Properties of BN Nanotubes with Intrinsic Defects," Physical Review B, Vol. 76, 2007, p. 155414.

[99] E. Hernandez, C. Goze, P. Bernier and A. Rubio, "Elastic Properties of Single-Wall Nanotubes," Applied Physics A, Vol. 68, No. 3, 1999, pp. 287-292.

[100] D. Srivastava, M. Menon and K.-J. Cho, "Anisotropic Nanomechanics of Boron Nitride Nanotubes: Nanostructured "Skin" Effect," Physical Review B, Vol. 63, 2001, p. 195413.

[101] T. Dumitrică and B. I. Yakobson, "Rate Theory of Yield in Boron Nitride Nanotubes," Physical Review B, Vol. 72, 2005, p. 035418.

[102] L. Chkhartishvili, "Quasi-Classical Theory of Substance Ground State," Technical University Press, Tbilisi, 2004.

[103] L. Chkhartishvili, "Quasi-Classical Method of Calculation of Substance Structural and Electronic Energy Spec- trum Parameters,” Tbilisi University Press, Tbilisi, 2006.

[104] L. Chkhartishvili, "Selection of Equilibrium Configurations for Crystalline and Molecular Structures Based on Quasi-Classical Inter-Atomic Potential," Transactions of the GTU, No. 427, 1999, pp. 13-19.

[105] L. Chkhartishvili, "On Quasi-Classical Estimations of Boron Nanotubes Ground-State Parameters," Journal of Physics: Conference Series, Vol. 176, 2009, p. 012013.

[106] L. Chkhartishvili, "Molar Binding Energy of the Boron Nanosystems," In: Proceedings of the 4th International Boron Symposium, Osmangazi University Press, Eskişehir, Turkey, 2009, pp. 153-160.

[107] L. Chkhartishvili, "Ground State Parameters of Wurtzite Boron Nitride: Quasi-Classical Estimations," In: Proceedings of the 1st International Boron Symposium, Dumlupinar University Press, Kütahya, 2002, pp. 139143.

[108] L. Chkhartishvili, "Boron Nitride Nanosystems of Regular Geometry," Journal of Physics: Conference Series, Vol. 176, 2009, p. 012014.

[109] L. S. Chkhartishvili, "Equilibrium Geometry of UltraSmall-Radius Boron Nitride Nanotubes," Material Science of Nanostructures, No. 1, 2009, pp. 33-44.

[110] Ch. Froese-Fischer, "The Hartree-Fock Method for Atoms: A Numerical Approach,” Wiley, New York, 1977.

[111] R. J. Boscovich, "Philosophiae Naturalis Theoria Redacta ad Inicam Legem Vitium in Natura Existentiam," Collegio Romano, Milano, 1758.

[112] T. L Hill and R. V. Chamberlin, "Fluctuations in Energy in Completely Open Small Systems," Nano Letters, Vol. 2, No. 6, 2002, pp. 609-613. 